\title{
CONTROLLABILITY OF SECOND ORDER SEMI-LINEAR NEUTRAL IMPULSIVE DIFFERENTIAL INCLUSIONS ON UNBOUNDED DOMAIN WITH INFINITE DELAY IN BANACH SPACES
}

\author{
Dimplekumar N. Chalishajar and Falguni S. Acharya
}

\begin{abstract}
In this paper, we prove sufficient conditions for controllability of second order semi-linear neutral impulsive differential inclusions on unbounded domain with infinite delay in Banach spaces using the theory of strongly continuous Cosine families. We shall rely on a fixed point theorem due to Ma for multi-valued maps. The controllability results in infinite dimensional space has been proved without compactness on the family of Cosine operators.
\end{abstract}

\section{Introduction}

In this paper we prove controllability of second order semi-linear neutral impulsive differential inclusions with non local conditions of the form:

$$
\left\{\begin{array}{l}
\frac{d}{d t}\left[y^{\prime}(t)-f\left(t, y_{t}, y^{\prime}(t)\right)\right] \in A y(t)+B u(t)+F\left(t, y_{t}, y^{\prime}(t)\right) ; \\
t \in J:=[0, \infty) ; t \neq t_{k}, \\
\left.\Delta y\right|_{t=t_{k}}=I_{k}\left(y_{t_{k}}, y^{\prime}\left(t_{k}\right)\right) ; k=1,2, \ldots, p \\
\left.\Delta y^{\prime}\right|_{t=t_{k}}=\overline{I_{k}}\left(y_{t_{k}}, y^{\prime}\left(t_{k}\right)\right) ; k=1,2, \ldots, p \\
y_{0}=\phi ; y^{\prime}(0)=x_{0},
\end{array}\right.
$$

where $A: D(A) \subset E \rightarrow E$ is the infinitesimal generator of a strongly continuous Cosine family $\{C(t): t \in \mathbb{R}\}$ defined on $E$, and $F: J \times \mathcal{B}_{h} \times E \rightarrow 2^{E}$ is a bounded, closed, convex multi-valued map. Let $J_{0}=(-\infty, 0]$ and non-local condition $\phi \in \mathcal{B}_{h}$ and $x_{0} \in E$ be the given initial values. Also, $f: J \times \mathcal{B}_{h} \rightarrow E$ is a given function, the state function $y(t)$ takes values in $E$, and the control $u \in L^{2}(J, U)$, a Banach space of admissible control functions with $U$ as a

Received December 18, 2009; Revised May 3, 2010.

2010 Mathematics Subject Classification. 34A37, 34G20, 34K20, 93B05, 93C10.

Key words and phrases. controllability, semi-linear neutral impulsive inclusions, convex multi-valued map, fixed point. 
Banach space. $B$ is a bounded linear operator from $U$ to $E$ and $E$ is a Banach space with norm $|\cdot|$.

Also, $0<t_{0}<t_{1}<\cdots<t_{p}<t_{p+1}=m(\rightarrow \infty$ as $t \rightarrow \infty) ; I_{k}, \overline{I_{k}} \in$ $C(E \times E, E), k=1,2, \ldots, p$ are bounded, $\left.\Delta y\right|_{t=t_{k}}=y\left(t_{k}^{+}\right)-y\left(t_{k}^{-}\right),\left.\Delta y^{\prime}\right|_{t=t_{k}}=$ $y^{\prime}\left(t_{k}^{+}\right)-y^{\prime}\left(t_{k}^{-}\right)$, and $y\left(t_{k}^{-}\right)$and $y\left(t_{k}^{+}\right), y^{\prime}\left(t_{k}^{-}\right)$and $y^{\prime}\left(t_{k}^{+}\right)$represent the left and right limits of $y(t)$ and $y^{\prime}(t)$ respectively, at $t=t_{k}$. Also, for any continuous function $y$ defined on the interval $J_{1}=(-\infty, \infty)$ with values in $E$ and for any $t \in J$, we denote by $y_{t}$ an element of $C\left(J_{0}, E\right)$ defined by $y_{t}(\theta)=y(t+\theta), \theta \in J_{0}$. Here we present the abstract space $\mathcal{B}_{h}$. Assume that $h:(-\infty, 0] \rightarrow(0, \infty)$ is a continuous function with $l:=\left(\int_{-\infty}^{0} h(s) d s<+\infty\right)$. Define

$\mathcal{B}_{h}=\{\phi:(-\infty, 0] \rightarrow E \mid$ for any $r>0, \phi(\theta)$ is bounded and measurable

$$
\text { function on } \left.[-r, 0] \text { and } \int_{-\infty}^{0} h(s) \sup _{s \leq \theta \leq 0}|\phi(\theta)| d s<+\infty\right\}
$$

where $\mathcal{B}_{h}$ endowed with the norm

$$
\|\phi\|_{\mathcal{B}_{h}}:=\int_{-\infty}^{0} h(s) \sup _{s \leq \theta \leq 0}|\phi(\theta)| d s \text { for all } \phi \in \mathcal{B}_{h} .
$$

Obviously, $\left(\mathcal{B}_{h},\|\cdot\|_{\mathcal{B}_{h}}\right)$ is a Banach space.

Lemma 1.1. Suppose $y \in \mathcal{B}_{h}$. Then for each $t \in J, y_{t} \in \mathcal{B}_{h}$. Moreover,

$$
l|y(t)| \leq\left\|y_{t}\right\|_{\mathcal{B}_{h}} \leq l \sup _{s \in[0, t]}\left(|y(s)|+\left\|y_{0}\right\|_{\mathcal{B}_{h}}\right),
$$

where $l:=\left(\int_{-\infty}^{0} h(s) d s<+\infty\right)$.

Proof. For a proof see [19].

The controllability of second-order systems with local and nonlocal conditions with impulse effect are interesting and researchers are engaged in it. A useful tool in the study of abstract second order equations is the theory of strongly continuous Cosine families ([26], [27]). Quinn and Carmichael [23] have first shown that the controllability problem in Banach spaces can be converted into a fixed point problem for a single valued map. Benchohra and Ntouyas [4] proved the existence and controllability results for nonlinear differential inclusions with nonlocal conditions. Also, they considered controllability of functional differential and integro-differential inclusions in Banach spaces [2] without infinite delay and impulse effect. In both the papers they used a fixed point theorem for condensing maps due to Martelli. George, Chalishajar, and Nandakumaran [12] studied the controllability on infinite time horizon for second-order semi-linear neutral functional differential inclusion in Banach spaces. Benchohra and Ntouyas [5] studied the existence of solutions for second order impulsive functional differential equation in Banach spaces using a fixed point theorem due to Schaefer [25] without infinite delay. Later, Liu [19] discussed controllability of impulsive neutral functional differential inclusion 
with infinite delay using the Martelli fixed point theorem; he considered first order systems with finite time horizon. However, in all of the above works, the authors imposed some severe compactness assumption on the operator family generated by $A$, which implies that $E$ has finite dimension. So the examples considered in $[19,2,4]$ are ordinary differential equations but not partial differential equations which shows a lack of existence (exact controllability) in abstract (control) space [28]. This fact and several other applications of neutral equation/inclusion are the main motivation of this paper.

On the other hand, the theory of impulsive differential equation has become an important area of investigation stimulated by their numerous applications to problem in mechanics, electric and electronic engineering, medicines, biology, ecology etc. Since many system arising from realistic models heavily depend on histories (which is characterized be the effect of infinite delay on state equations), there is a need to study partial functional differential systems/inclusions with infinite delay (also on unbounded time interval). Many evolution processes are characterized by the fact that at certain moments of time they experience a change of state abruptly. This process is subject to short term perturbation whose duration is negligible in comparison with the duration of the process [24].

In this paper, we prove sufficient conditions for controllability of secondorder semi-linear neutral impulsive differential inclusions in Banach spaces with infinite delay and unbounded domain using the theory of strongly continuous Cosine families. We shall rely on a fixed point theorem due to Ma for multivalued maps [20]. This work is the generalization of papers of Benchohra and Ntouyas [5], George, Chalishajar and Nandakumaran [12] and Liu [19].

After preliminaries in Section 2, we reduce the controllability problem (1.1) to the search for fixed points of a suitable multi-valued map on $C(J, E)$ in Section 3. In order to prove the existence of fixed points in finite dimension, we shall rely on a theorem due to Ma [20], which is an extension of Schaefer's theorem [25] to multi-valued maps between locally convex topological spaces. An example is provided to support the theory in Section 4. Section 5 deals with exact controllability of system (1.1) in infinite dimension spaces without having a compactness assumption on family of Cosine operators.

\section{Preliminaries}

In this section, we introduce notations, definitions, and preliminary facts from multi-valued analysis which are used throughout this paper. Let $J_{m}=$ $[0, m], m \in \mathbb{N}$. The space $C(J, E)$ is the Banach space of continuous functions from $J$ into $E$ with the metric [11]

$$
d(y, z)=\sum_{m=0}^{\infty} \frac{2^{-m}\|y-z\|_{m}}{1+\|y-z\|_{m}} \text { for each } y, z \in C(J, E)
$$


where

$$
\|y\|_{m}:=\sup \left\{|y(t)|: t \in J_{m}\right\} .
$$

Let $B(E)$ be the Banach space of bounded linear operators from $E$ to $E$ with the standard norm. A measurable function $y: J \rightarrow E$ is Bochner integrable if and only if $|y|$ is Lebesgue integrable. For properties of the Bochner integral, we refer to [6]. Let $L^{1}(J, E)$ denotes the Banach space of Bochner integrable functions and $U_{b}$ denotes a neighborhood of 0 in $C(J, E)$ defined by

$$
U_{b}:=\left\{y \in C(J, E):\|y\|_{m} \leq b\right\} .
$$

The convergence in $C(J, E)$ is the uniform convergence in the compact intervals, i.e., $y_{j} \rightarrow y$ in $C(J, E)$ if and only if $\left\|y_{j}-y\right\|_{m} \rightarrow 0$ in $C\left(J_{m}, E\right)$ as $j \rightarrow \infty$ for each $m \in \mathbb{N}$. A set $M \subseteq C(J, E)$ is a bounded set if and only if there exists a positive function $\xi \in C\left(J, \mathbb{R}_{+}\right)$such that

$$
|y(t)| \leq \xi(t) \text { for all } t \in J \text { and } y \in M .
$$

The Arzela-Ascoli theorem says that a set $M \subseteq C(J, E)$ is compact if and only if $M$ is in the Banach space $\left(C\left(J_{m}, E\right),\|\cdot\|_{m}\right)$.

We say that one-parameter family $\{C(t): t \in \mathbb{R}\}$ of bounded linear operators in $B(E)$ is a strongly continuous Cosine family if and only if

(1) $C(0)=I, I$ is the identity operator on $E$.

(2) $C(t+s)+C(t-s)=2 C(t) C(s)$ for all $s, t \in \mathbb{R}$.

(3) the map $t \mapsto C(t) y$ is strongly continuous in $t$ on $\mathbb{R}$ for each fixed $y \in E$.

The strongly continuous Sine family $\{S(t): t \in \mathbb{R}\}$, associated to the strongly continuous Cosine family $\{C(t): t \in \mathbb{R}\}$ is defined by

$$
S(t) y=\int_{0}^{t} C(s) y d s, y \in E, t \in \mathbb{R} .
$$

Assume the following condition on $A$.

(H1) $A$ is the infinitesimal generator of a strongly continuous Cosine family $C(t), t \in \mathbb{R}$, which is compact for $t>0$; of bounded linear operators $E$ into itself and the adjoint operator $A^{*}$ is densely defined, i.e., $\overline{D\left(A^{*}\right)}=E^{*}$ (see [6]).

The infinitesimal generator of a strongly continuous Cosine family $C(t), t \in$ $\mathbb{R}$ is the operator $A: D(A) \subset E \rightarrow E$ defined by

$$
A y=\left.\frac{d^{2}}{d t^{2}} C(t) y\right|_{t=0}, y \in D(A),
$$

where $D(A)=\left\{y \in E: C(\cdot) y \in C^{2}(\mathbb{R}, E)\right\}$, endowed with the norm

$$
\|y\|_{E}=\|y\|+\|A y\|, y \in D(A) .
$$


Define $E_{1}=\left\{y \in E: C(\cdot) y \in C^{1}(\mathbb{R}, E)\right\}$. It was proved by Kisynski [17] that $E_{1}$ endowed with the norm

$$
\|y\|_{1}=\|y\|+\sup _{0 \leq t \leq 1}\|A S(t) y\|, y \in E
$$

is a Banach space. The operator valued function $G(t)=\left(\begin{array}{cc}C(t) & S(t) \\ A S(t) & A C(t)\end{array}\right)$ is a strongly continuous group of linear operators on the space $E_{1} \times E$ generated by the operator $\widetilde{A}=\left(\begin{array}{ll}0 & I \\ A & 0\end{array}\right)$ defined on $D(A) \times E_{1}$. From this, it follows that $A S(t): E_{1} \rightarrow E$ is a bounded linear operator and that $A S(t) \rightarrow 0$ as $t \rightarrow 0$ for each $y \in E_{1}$.

Lemma 2.1 ([26]). Let (H1) hold. Then the following hold.

(1) There exist constants $M_{1} \geq 1$ and $w \geq 0$ such that

$$
|C(t)| \leq M_{1} e^{w|t|} \text { and }\left|S(t)-S\left(t^{*}\right)\right| \leq M_{1} \int_{0}^{t^{*}} e^{w|s|} d s \text { for } t, t^{*} \in \mathbb{R} \text {. }
$$

(2) For $y \in E, S(t) y \in E_{1}$ and so $S(t) E \subset E_{1}$ for $t \in \mathbb{R}$.

(3) For $y \in E_{1}, C(t) y \in E_{1}, S(t) y \in D(A)$ and $\frac{d}{d t} C(t) y=A S(t) y, t \in \mathbb{R}$.

(4) For $y \in D(A), C(t) y \in D(A)$ and $\frac{d^{2}}{d t^{2}} C(t) y=A C(t) y$ for $t \in \mathbb{R}$.

Lemma $2.2([26])$. Let $(\mathrm{H} 1)$ holds; let $v \in C^{1}(\mathbb{R}, E)$, and let

$$
q(t)=\int_{0}^{t} S(t-s) v(s) d s .
$$

Then,

$$
q \in C^{2}(\mathbb{R}, E) \text { for } t \in \mathbb{R} \text {, and } q(t) \in D(A) .
$$

Further, $q$ satisfies

$$
q^{\prime}(t)=\int_{0}^{t} C(t-s) v(s) d s \quad \text { and } \quad q^{\prime \prime}(t)=A q(t)+v(t) .
$$

For more details on strongly continuous Cosine and Sine family, we refer the reader to the book of Goldstein [13].

We now recall some preliminaries about multi-valued maps. Let $(X,\|\cdot\|)$ be a Banach space. A multi-valued map $G: X \rightarrow 2^{X}$ is convex (resp. closed) if $G(x)$ is convex (resp. closed) in $X$ for all $x \in X$. The map $G$ is bounded on bounded sets if $G(B)=U_{x \in B} G(x)$ is bounded in $X$ for any bounded set $B$ of $X$ (i.e. $\sup _{x \in B}\{\sup \{\|y\|: y \in G(x)\}\}<\infty$ ). $G$ is called upper semi continuous (u.s.c.) on $X$ if for each $x_{0} \in X$ the set $G\left(x_{0}\right)$ is a nonempty, closed subset of $X$ and if for each open set $B$ of $X$ containing $G\left(x_{0}\right)$, there exists an open neighborhood $A$ of $x_{0}$ such that $G(A) \subseteq B$. The map $G$ is said to be completely continuous if $G(B)$ is relatively compact for every bounded subset $B \subseteq X$.

If the multi-valued map $G$ is completely continuous with nonempty compact values, then $G$ is u.s.c. if and only if $G$ has a closed graph. That is, if $x_{n} \rightarrow x_{0}$ and $y_{n} \rightarrow y_{0}$, where $y_{n} \in G\left(x_{n}\right)$, then $y_{0} \in G\left(x_{0}\right)$. We say, $G$ has a fixed point 
if there is $x \in X$ such that $x \in G(x)$. In the following, $B C C(X)$ denotes the set of all nonempty bounded, closed and convex subsets of $X$.

A multi-valued map $G: J \rightarrow B C C(X)$ is said to be measurable, if for each $x \in X$, the distance function $Y: J \rightarrow \mathbb{R}$ defined by

$$
Y(t)=d(x, G(t))=\inf \{|x-z|: z \in G(t)\}
$$

is measurable. For more details on multi-valued maps, see ([10], [16]).

An upper semi-continuous map $G: X \rightarrow 2^{X}$ is said to be condensing, if for any subset $B \subseteq X$, with $\alpha(B) \neq 0$, we have $\alpha(G(B))<\alpha(B)$, where $\alpha$ denotes the Kuratowski measure of non-compactness.

We remark that a completely continuous multi-valued map is the easiest example of a condensing map. For more details on multivalued maps see the book of Deimling [10].

We assume the following hypotheses:

(H2) Let $m \in \mathbb{N}$. Let $W: L^{2}(J, U) \rightarrow E$ be the linear operator defined by

$$
W u=\int_{0}^{m} S(m-s) B u(s) d s .
$$

Then $W: L^{2}(J, U) /$ ker $W \rightarrow E$ induces a bounded invertible operator $\widetilde{W}^{-1}$ and there exist positive constants $M_{2}$ and $M_{3}$ such that $|B| \leq M_{2}$ and $\left|\widetilde{W}^{-1}\right| \leq$ $M_{3}$. For construction of $\widetilde{W}^{-1}$, refer to [23].

(H3) The function $f: J \times \mathcal{B}_{h} \times E \rightarrow E$ is completely continuous and for any bounded set $Q \subseteq C\left(J_{1}, E\right)$, the family $\left\{t \mapsto f\left(t, y_{t}\right): y \in Q\right\}$ is equi-continuous in $C(J, E)$.

Further, assume that there exist constants $0 \leq c_{1}<1$ and $c_{2} \geq 0$ such that for all $t \in J, \phi \in \mathcal{B}_{h}$, we have

$$
|f(t, \phi, y)| \leq c_{1}\left(\|\phi\|_{\mathcal{B}_{h}}+|y|\right)+c_{2} .
$$

(H4)(i) The multi-valued map $(t, \psi, y) \mapsto F(t, \psi, y)$ is measurable with respect to $t$ for each $\psi \in \mathcal{B}_{h}$ and $y \in E$ and $F$ is u.s.c. with respect to second and third variable for each $t \in J$. Moreover, for each fixed $z \in C\left(J_{1}, E\right)$ and $y \in C(J, E)$, the set

$$
S_{F, z, y}=\left\{v \in L^{1}(J, E): v(t) \in F\left(t, z_{t}, y(t)\right) \text { for a.e. } t \in J\right\}
$$

is nonempty.

(ii) We assume $F$ satisfies the following estimate. Given $\psi \in \mathcal{B}_{h}$ and $y \in E$, there exists $P \in L^{1}\left(J, \mathbb{R}^{+}\right)$

$$
\|F(t, \psi, y)\|:=\sup \{|v|: v \in F(t, \psi, y)\} \leq P(t) \Omega\left(\|\psi\|_{\mathcal{B}_{h}}+|y|\right),
$$

where $\Omega: \mathbb{R}^{+} \rightarrow(0, m]$ is continuous and increasing and there is a $c>0$ such that the integral $\int_{c}^{\infty} \frac{d s}{s+\psi(s)}$ is sufficiently large (an explicit lower bound and expression for $c$ can be given). 
(H5)(i) Each function $I_{k}: \mathcal{B}_{h} \times E \rightarrow E_{1}$ is completely continuous and there exist constants $c_{k}^{j}, j=1,2$ such that

$\left\|I_{k}(\psi, y)\right\|_{E_{1}} \leq c_{k}^{1}\left(\|\psi\|_{\mathcal{B}_{h}}+|y|\right)+c_{k}^{2} ; k=1,2, \ldots, p$ for every $(\psi, y) \in \mathcal{B}_{h} \times E$.

(ii) The functions $\overline{I_{k}}: \mathcal{B}_{h} \times E \rightarrow E$ are completely continuous and there exist constants $d_{k}^{j}, j=1,2$ such that

$\left\|\overline{I_{k}}(y)\right\|_{E} \leq d_{k}^{1}\left(\|\psi\|_{\mathcal{B}_{h}}+|y|\right)+d_{k}^{2} ; k=1,2, \ldots, p$ for every $(\psi, y) \in \mathcal{B}_{h} \times E$.

(H6) For each bounded set $Q \subseteq C((-\infty, m], E)$ and for each $t \in J_{m}$ the set

$$
\begin{aligned}
& \left\{C(t) \phi(0)+S(t)\left[x_{0}-f(0, \phi)\right]+\int_{0}^{t} C(t-s) f\left(s, y_{s}, y^{\prime}(s)\right) d s\right. \\
& \quad+\int_{0}^{t} S(t-s) B u(s) d s+\int_{0}^{t} S(t-s) v(s) d s \\
& \left.\quad+\sum_{0<t_{k}<t} C\left(t-t_{k}\right) I_{k}\left(y_{t_{k}}, y^{\prime}\left(t_{k}\right)\right)+\sum_{0<t_{k}<t} S\left(t-t_{k}\right) \overline{I_{k}}\left(y_{t_{k}}, y^{\prime}\left(t_{k}\right)\right): y \in Q\right\}
\end{aligned}
$$

is relatively compact in $E$.

The integral equation formulation of the system (1.1) is given by

$$
\left\{\begin{aligned}
y(t)= & \phi(t) ; \quad \text { if } \quad t \in J_{0} \\
y(t)= & C(t) \phi(0)+S(t)\left[x_{0}-f\left(0, \phi, x_{0}\right)\right]+\int_{0}^{t} C(t-s) f\left(s, y_{s}, y^{\prime}(s)\right) d s \\
& +\int_{0}^{t} S(t-s) B u(s) d s+\int_{0}^{t} S(t-s) v(s) d s+\sum_{0<t_{k}<t} C\left(t-t_{k}\right) \\
& I_{k}\left(y_{t_{k}}, y^{\prime}\left(t_{k}\right)\right)+\sum_{0<t_{k}<t} S\left(t-t_{k}\right) \overline{I_{k}}\left(y_{t_{k}}, y^{\prime}\left(t_{k}\right)\right) ; \quad \text { if } \quad t \in J
\end{aligned}\right.
$$

(see [21]), where $v \in S_{F, y, y^{\prime}}=\left\{v \in L^{1}(J, E): v(t) \in F\left(t, y_{t}, y^{\prime}(t)\right)\right.$ for a.e. $t \in J\}$ is called the mild solution on $J$ of the inclusion (1.1) provided $\int_{0}^{t} C(t-$ $s) f\left(s, y_{s}, y^{\prime}(s)\right) d s$ is integrable, $x_{0}=\phi \in \mathcal{B}_{h}$.

Remark 2.3. If $\operatorname{dim} E<\infty$ and $J$ is a compact real interval, then $S_{F, y, y^{\prime}} \neq \phi$ (see [18]).

Definition 2.4. The system (1.1) is said to be controllable on $J^{\prime}=J /\left\{t_{1}, t_{2}\right.$, $\left.\ldots, t_{p}\right\}$ if for every $\phi \in \mathcal{B}_{h}$ with $\phi(0) \in D(A), x_{0} \in E_{1}, y_{1} \in E$ and there exists a control $u \in L^{2}\left(J_{m}, U\right)$ such that the solution $y(\cdot)$ of $(1.1)$ satisfies $y(m)=y_{1}$ and the conditions $\left.\Delta y\right|_{t=t_{k}}=I_{k}\left(y_{t_{k}}, y^{\prime}\left(t_{k}\right)\right)$ and $\left.\Delta y^{\prime}\right|_{t=t_{k}}=\overline{I_{k}}\left(y_{t_{k}}, y^{\prime}\left(t_{k}\right)\right) ; k=$ $1,2, \ldots, p$ and $y^{\prime}(0)=x_{0}$.

Lemma 2.5 ([18]). Let $J$ be a compact real interval and $X$ be a Banach space. Let $F$ be a multi-valued map satisfying (H4)(ii) and let $\Gamma$ be a linear continuous mapping from $L^{1}(J, X)$ to $C(J, X)$. Then the operator

$\Gamma o S_{F}: C(J, X) \rightarrow B C C(C(J, X))$ defined by $y \rightarrow\left(\Gamma o S_{F}\right)(y):=\Gamma\left(S_{F, y_{t}, y^{\prime}}\right)$ is a closed graph operator. 
Lemma 2.6 ([20]). Let $X$ be a locally convex space and $N_{1}: X \rightarrow 2^{X}$ be a compact, convex, u.s.c. multi-valued map such that there exists a closed neighborhood $U_{p}$ of 0 for which $N_{1}\left(U_{p}\right)$ is a relatively compact set for each neighborhood $U_{p}$. If the set

$$
\Omega_{1}:=\left\{y \in X: \lambda y \in N_{1}(y) \text { for some } \lambda>1\right\}
$$

is bounded, then $N_{1}$ has a fixed point.

\section{Controllability result}

We now state and prove the main controllability result.

Theorem 3.1. Assume that the hypotheses (H1)-(H6) are satisfied. Then the inclusion (1.1) is controllable for $x_{0}$ and $y_{1}$ on $J$.

Proof. Consider the space

$$
\begin{gathered}
\mathcal{B}_{b}\left(J_{1}, E\right)=\left\{y:(-\infty, m) \rightarrow E ; y(\mathrm{t}) \text { is continuous at } t \neq t_{k}, y\left(t_{k}^{-}\right)=y\left(t_{k}\right),\right. \\
\text { and } \left.y\left(t_{k}^{+}\right) \text {exists for every } k=1,2, \ldots, p\right\}
\end{gathered}
$$

and

$$
\begin{gathered}
\mathcal{B}_{b}^{1}\left(J_{1}, E\right)=\left\{y \in \mathcal{B}_{b}\left(J_{1}, E\right): y^{\prime}(t) \text { is continuous at } t \neq t_{k}, y^{\prime}\left(t_{k}^{-}\right)=y^{\prime}\left(t_{k}\right),\right. \\
\text { and } \left.y^{\prime}\left(t_{k}^{+}\right) \text {exists for every } k=1,2, \ldots, p\right\}
\end{gathered}
$$

with the norm

$$
\|y\|_{b^{1}}=\left\|y_{0}\right\|_{\mathcal{B}_{h}}+\sup _{t \in J_{m}}\left\{\|y(s)\|_{b},\left\|y^{\prime}(s)\right\|_{b}\right\} ; 0 \leq s \leq b, y \in \mathcal{B}_{b} \text { and } y^{\prime} \in \mathcal{B}_{b}^{1},
$$

where $\|y\|_{b}=\sup _{t \in J_{m}}|y(t)|$. Here, $y_{k}$ is a restriction of $y$ to $J_{k}=\left(t_{k}, t_{k+1}\right] ; k=$ $1,2, \ldots, p$ such that $\left\|y_{k}\right\|_{J_{k}}=\sup _{s \in J_{k}}\left\|y_{k}(s)\right\|$.

To consider the impulsive conditions, it is convenient to introduce some additional notations. In what follows, put $t_{0}=0, t_{p+1}=m$ and for $y \in \mathcal{B}_{b}$ we denote by $\widetilde{y_{k}} \in C\left(\left(t_{k}, t_{k+1}\right] ; E\right), k=0,1, \ldots, p$, the function given by

$$
\widetilde{y_{k}}(t)=\left\{\begin{array}{l}
y(t) ; t \in\left(t_{k}, t_{k+1}\right] \\
y\left(t_{k}^{+}\right) ; t=t_{k} .
\end{array}\right.
$$

Let us define a space

$$
Z=\left\{\mathcal{B}_{b}\left(J_{1}, E\right) \cap \mathcal{B}_{b}^{1}\left(J_{1}, E\right) \cap C^{2}\left(J_{1}, E\right)\right\} .
$$


CONTROLLABILITY OF SECOND ORDER SEMI-LINEAR NEUTRAL IMPULSIVE 821

Using the hypothesis (H2) for $y \in Z$, we define the control formally as

$$
\begin{aligned}
u(t)=\widetilde{W}^{-1}[ & y_{1}-C(m) \phi(0)-S(m)\left[x(0)-f\left(0, \phi, x_{0}\right)\right] \\
& -\int_{0}^{m} C(m-s) f\left(s, y_{s}, y^{\prime}(s)\right) d s-\int_{0}^{m} S(m-s) v(s) d s \\
& -\sum_{0<t_{k}<t} C\left(t-t_{k}\right) I_{k}\left(y_{t_{k}}, y^{\prime}\left(t_{k}\right)\right) \\
& \left.-\sum_{0<t_{k}<t} S\left(t-t_{k}\right) \overline{I_{k}}\left(y_{t_{k}}, y^{\prime}\left(t_{k}\right)\right)\right](t) .
\end{aligned}
$$

Let $h$ be given by

$$
\begin{aligned}
h(t)= & C(t) \phi(0)+S(t)\left[x_{0}-f\left(0, \phi, x_{0}\right)\right]+\int_{0}^{t} C(t-s) f\left(s, y_{s}, y^{\prime}(s)\right) d s \\
& +\int_{0}^{t} S(t-s) v(s) d s+\int_{0}^{t} S(t-\eta) B u(\eta) d \eta \\
& +\sum_{0<t_{k}<t} C\left(t-t_{k}\right) I_{k}\left(y_{t_{k}}, y^{\prime}\left(t_{k}\right)\right) \\
& +\sum_{0<t_{k}<t} S\left(t-t_{k}\right) \overline{I_{k}}\left(y_{t_{k}}, y^{\prime}\left(t_{k}\right)\right) .
\end{aligned}
$$

Using the above control, define a multi-valued map $N_{1}: Z \rightarrow 2^{Z}$ by

$$
\left(N_{1} y\right)(t)=\phi(t) \text { for }-r \leq t \leq 0 ; r \in \mathbb{N},
$$

and for $m \geq t \geq 0$,

$$
N_{1} y:=\left\{h \in C\left(J_{k}, E\right): h \text { satisfies }(3.2)\right\} .
$$

Here, $u$ is defined as in (3.1) and $v \in S_{F, y_{t}, y^{\prime}}$.

Clearly, the fixed points of $N_{1}$ are mild solutions to (1.1). We show that $N_{1}$ satisfies the hypotheses of Lemma 2.4.

Clearly, $\left(N_{1} y\right)(m)=y_{1}$, which means that the control $u$ steers the system from initial state $y_{0}$ to $y_{1}$ in time $m$, provided we obtain a fixed point of the nonlinear operator $N_{1}$.

Step 1: The set $\Psi:=\left\{y \in Z: \lambda y \in N_{1}(y), \lambda>1\right\}$ is bounded. To see this, let $y \in \Psi$, then $y$ has the representation for $t \geq 0$.

$$
\begin{aligned}
y(t)= & \lambda^{-1} h(t) \\
= & \lambda^{-1} C(t) \phi(0)+\lambda^{-1} S(t)\left[x_{0}-f\left(0, \phi, x_{0}\right)\right] \\
& +\lambda^{-1} \int_{0}^{t} C(t-s) f\left(s, y_{s}, y^{\prime}(s)\right) d s \\
& +\lambda^{-1} \int_{0}^{t} S(t-s) v(s) d s+\lambda^{-1} \int_{0}^{t} S(t-\eta) B u(\eta) d \eta
\end{aligned}
$$




$$
+\lambda^{-1}\left[\sum_{0<t_{k}<t} C\left(t-t_{k}\right) I_{k}\left(y_{t_{k}}, y^{\prime}\left(t_{k}\right)\right)+\sum_{0<t_{k}<t} S\left(t-t_{k}\right) \overline{I_{k}}\left(y_{t_{k}}, y^{\prime}\left(t_{k}\right)\right)\right],
$$

where $u$ is defined as in (3.1).

It is then easy to observe that $y$ is a mild solution of the inclusion (1.1).

Thus, we have to obtain bounds on $y$ and $y^{\prime}$ independent of $\lambda>1$ which will prove the boundedness of $\Psi$.

$$
\begin{aligned}
|y(t)| \leq & M|\phi|+M m\left[\left|x_{0}\right|+c_{1}\left(\|\phi\|_{\mathcal{B}_{h}}+\left|x_{0}\right|\right)+c_{2}\right] \\
& +M \int_{0}^{t}\left\{c_{1}\left(|| y_{s}||+\left|y^{\prime}(s)\right|\right)+c_{2}\right\} d s \\
& +M m \int_{0}^{t} P(s) \Omega\left(\left\|y_{s}\right\|+\left|y^{\prime}(s)\right|\right) d s \\
& +M m M_{2} M_{3} \int_{0}^{t}\left\{\left|y_{1}\right|+M|\phi|+M m\left[\left|x_{0}\right|+c_{1}\left(|| \phi||+\left|x_{0}\right|\right)+c_{2}\right]\right. \\
& +M \int_{0}^{m}\left\{c_{1}\left(\left\|y_{\eta}\right\|+\left|y^{\prime}(\eta)\right|\right)+c_{2}\right\} d \eta \\
& \left.+M m \int_{0}^{m} P(\eta) \Omega\left(\left\|y_{\eta}\right\|+\left|y^{\prime}(\eta)\right|\right) d \eta\right\} d s \\
& +M \sum_{0<t_{k}<t}\left\{c_{k}^{1}\left(\left\|y_{t_{k}}\right\|+\left|y^{\prime}\left(t_{k}\right)\right|\right)+c_{k}^{2}\right\} \\
& +M m \sum_{0<t_{k}<t}\left\{d_{k}^{1}\left(\left\|y_{t_{k}}\right\|+\left|y^{\prime}\left(t_{k}\right)\right|\right)+d_{k}^{2}\right\} ; t \in J_{m} .
\end{aligned}
$$

Using the assumptions, it is easy to obtain positive constants $C_{1}, C_{2}, C_{3}$ depending on the initial values and bounds on the Cosine and Sine operators such that

$$
\begin{aligned}
|y(t)| \leq & C_{1}+C_{2} \int_{0}^{t}\left(\left\|y_{s}\right\|+\left|y^{\prime}(s)\right|\right) d s+C_{3} \int_{0}^{t} P(s) \Omega\left(\left\|y_{s}\right\|+\left|y^{\prime}(s)\right|\right) d s \\
& +M \sum_{0<t_{k}<t}\left\{c_{k}^{1}\left(\left\|y_{t_{k}}\right\|+\left|y^{\prime}\left(t_{k}\right)\right|\right)+c_{k}^{2}\right\} \\
& +M m \sum_{0<t_{k}<t}\left\{d_{k}^{1}\left(\left\|y_{t_{k}}\right\|+\left|y^{\prime}\left(t_{k}\right)\right|\right)+d_{k}^{2}\right\} .
\end{aligned}
$$

Denoting by $v(t)$ the right-hand side of the above inequality, we get

$$
\mu(t) \leq v(t) .
$$

Here the function $\mu$ is defined by

$$
\mu(t)=\sup \{|y(s)|:-r \leq s \leq t\} ; t \in J_{m}, r \in \mathbb{N} .
$$


CONTROLLABILITY OF SECOND ORDER SEMI-LINEAR NEUTRAL IMPULSIVE 823

Further, $v(0)=C_{1}$ and

$$
\begin{aligned}
v^{\prime}(t) & \leq C_{2}\left(\mu(t)+\left|y^{\prime}(t)\right|\right)+C_{3} P(t) \Omega\left(\mu(t)+\left|y^{\prime}(t)\right|\right) \\
& \leq C_{2}\left(v(t)+\left|y^{\prime}(t)\right|\right)+C_{3} P(t) \Omega\left(v(t)+\left|y^{\prime}(t)\right|\right), t \in J_{m} .
\end{aligned}
$$

Now,

$$
\begin{aligned}
y^{\prime}(t)= & \lambda^{-1} A S \phi(0)+\lambda^{-1} C(t)\left[x_{0}-f\left(0, \phi, x_{0}\right)\right]+\lambda^{-1} f\left(t, y_{t}, y^{\prime}(t)\right) \\
& +\lambda^{-1} \int_{0}^{t} A S(t-s) f\left(s, y_{s}, y^{\prime}(s)\right) d s+\lambda^{-1} \int_{0}^{t} C(t-\eta) B \widetilde{W}^{-1} \\
& {\left[y_{1}-C(m) \phi(0)-S(m)\left[x_{0}-f\left(0, \phi, x_{0}\right)\right]-\int_{0}^{m} C(m-s)\right.} \\
& \left.f\left(s, y_{s}, y^{\prime}(s)\right) d s-\int_{0}^{m} S(m-s) v(s) d s\right](\eta) d \eta+\lambda^{-1} \int_{0}^{t} C(t-s) v(s) d s \\
& +\sum_{0<t_{k}<t} A S\left(t-t_{k}\right) I_{k}\left(y_{t_{k}}, y^{\prime}\left(t_{k}\right)\right)+\sum_{0<t_{k}<t} C\left(t-t_{k}\right) \overline{I_{k}}\left(y_{t_{k}}, y^{\prime}\left(t_{k}\right)\right) .
\end{aligned}
$$

We can estimate $y^{\prime}$ in a similar fashion. There exist positive constants $C_{4}, C_{5}$, $C_{6}, C_{7}$ such that

$$
\begin{aligned}
\left|y^{\prime}(t)\right| \leq & C_{4}+C_{5}\left(\left\|y_{t}\right\|+\left|y^{\prime}(t)\right|\right)+C_{6} \int_{0}^{t}\left(\left\|y_{s}\right\|+\left|y^{\prime}(t)\right|\right) d s \\
& +C_{7} \int_{0}^{t} P(s) \Omega\left(\left\|y_{s}\right\|+\left|y^{\prime}(s)\right|\right) d s \\
\leq & C_{4}+C_{5} \mu(t)+C_{6} \int_{0}^{t}\left\|y_{s}\right\| d s+C_{7} \int_{0}^{t} P(s) \Omega\left(\left\|y_{s}\right\|+\left|y^{\prime}(s)\right|\right) d s \\
\leq & C_{4}+C_{5} v(t)+C_{6} \int_{0}^{t}\left\|y_{s}\right\| d s+C_{7} \int_{0}^{t} P(s) \Omega\left(\left\|y_{s}\right\|+\left|y^{\prime}(s)\right|\right) d s .
\end{aligned}
$$

Denoting by $r(t)$ the right-hand side of the above inequality, we have

$$
\begin{aligned}
\left|y^{\prime}(t)\right| & \leq r(t), t \in J \\
r(0) & =C_{4}+C_{5} C_{1}
\end{aligned}
$$

and

$$
\begin{aligned}
r^{\prime}(t) & \leq C_{5} v^{\prime}(t)+C_{6} \mu(t)+C_{7} P(t) \Omega\left(\mu(t)+\left|y^{\prime}(t)\right|\right) \\
& \leq C_{5} v^{\prime}(t)+C_{6} v(t)+C_{7} P(t) \Omega(v(t)+r(t)) \\
& \leq\left(C_{2} C_{5}+C_{6}\right) v(t)+\left(C_{3} C_{5}+C_{7}\right) P(t) \Omega(v(t)+r(t))
\end{aligned}
$$

where the last inequality is obtained from the estimate of $v^{\prime}(t)$. Let

$$
w(t)=v(t)+r(t), t \in J .
$$

Then,

and

$$
c:=w(0)=v(0)+r(0)=C_{1}+C_{4}+C_{1} C_{5}
$$

$$
w^{\prime}(t)=v^{\prime}(t)+r^{\prime}(t)
$$




$$
\begin{aligned}
& \leq\left(C_{2}+C_{2} C_{5}+C_{6}\right) v(t)+\left(C_{3}+C_{3} C_{5}+C_{7}\right) P(t) \Omega(v(t)+r(t)) \\
& =\left(C_{2}+C_{2} C_{5}+C_{6}\right) w(t)+\left(C_{3}+C_{3} C_{5}+C_{7}\right) P(t) \Omega(w(t)) \\
& \leq m(t)[w(t)+\Omega(w(t))],
\end{aligned}
$$

where $m(t):=\max \left\{C_{2}+C_{2} C_{5}+C_{6},\left(C_{3}+C_{3} C_{5}+C_{7}\right) P(t)\right\}$. This implies that

$$
\int_{c}^{w(t)} \frac{d s}{s+\Omega(s)}=\int_{w(0)}^{w(t)} \frac{d s}{s+\Omega(s)} \leq \int_{0}^{m} m(s) d s<\int_{c}^{\infty} \frac{d s}{s+\Omega(s)},
$$

where the last inequality follows from assumption (H4)(ii). This implies that there exists a constant $L$ such that

$$
w(t)=v(t)+r(t) \leq L, t \in J
$$

Thus

$$
\begin{aligned}
\|y(t)\| & \leq v(t) \leq L, t \in J \\
\left\|y^{\prime}(t)\right\| & \leq r(t) \leq L, t \in J
\end{aligned}
$$

and hence $\Psi$ is bounded.

Step 2: $N_{1} y$ is convex for each $y \in Z$.

Indeed, if $h_{1}, h_{2} \in N_{1} y$, then there exist $v_{1}, v_{2} \in S_{F_{i}, y_{t}, y^{\prime}}$ such that for $i=1,2$, we have

$$
\begin{aligned}
h_{i}(t)= & C(t) \phi(0)+S(t)\left[x_{0}-f\left(0, \phi, x_{0}\right)\right]+\int_{0}^{t} C(t-s) f\left(s, y_{s}, y^{\prime}(s)\right) d s \\
& +\int_{0}^{t} S(t-s) v_{i}(s) d s+\int_{0}^{t} S(t-\eta) B u(\eta) d \eta \\
& +\sum_{0<t_{k}<t} C\left(t-t_{k}\right) I_{k}\left(y\left(t_{k}\right), y^{\prime}\left(t_{k}\right)\right)+\sum_{0<t_{k}<t} S\left(t-t_{k}\right) \overline{I_{k}}\left(y\left(t_{k}\right), y^{\prime}\left(t_{k}\right)\right),
\end{aligned}
$$

where $u$ is defined as in (3.1) with $v$ replaced by $v_{i}$. Then it is an easy matter to see that, for $0 \leq k \leq 1$,

$$
\begin{aligned}
& \left(k h_{1}+(1-k) h_{2}\right)(t) \\
= & C(t) \phi(0)+S(t)\left[x_{0}-f\left(0, \phi, x_{0}\right)\right]+\int_{0}^{t} C(t-s) f\left(s, y_{s}, y^{\prime}(s)\right) d s \\
& +\int_{0}^{t} S(t-s)\left(k v_{1}+(1-k) v_{2}\right)(s) d s+\int_{0}^{t} S(t-\eta) B u(\eta) d \eta \\
& +\sum_{0<t_{k}<t} C\left(t-t_{k}\right) I_{k}\left(y\left(t_{k}\right), y^{\prime}\left(t_{k}\right)\right)+\sum_{0<t_{k}<t} S\left(t-t_{k}\right) \overline{I_{k}}\left(y\left(t_{k}\right), y^{\prime}\left(t_{k}\right)\right),
\end{aligned}
$$

where $u$ is defined as in (3.1) with $v=k v_{1}+(1-k) v_{2}$.

Since $S_{F, y_{t}, y^{\prime}}$ is convex as $F$ is convex, we have $v=k v_{1}+(1-k) v_{2} \in S_{F, y_{t}, y^{\prime}}$ and hence $k h_{1}+(1-k) h_{2} \in N_{1} y$.

Step 3: $N_{1}\left(U_{q}\right)$ is bounded in $Z$ for each $q \in \mathbb{N}$, where $U_{q}$ is a neighborhood of 0 in $Z$. 
CONTROLLABILITY OF SECOND ORDER SEMI-LINEAR NEUTRAL IMPULSIVE 825

We have to show that there exists a positive constant $l_{1}$ such that for any $y \in U_{q}$ and $h \in N_{1} y$ such that $\|h\|_{Z} \leq l_{1}$. In other words, we have to bound the sup-norm of both $h$ and $h^{\prime}$. We can write

$$
\begin{aligned}
h(t)= & C(t) \phi(0)+S(t)\left[x_{0}-f\left(0, \phi, x_{0}\right)\right]+\int_{0}^{t} C(t-s) f\left(s, y_{s}, y^{\prime}(s)\right) d s \\
& +\int_{0}^{t} S(t-s) v(s) d s+\int_{0}^{t} S(t-\eta) B u(\eta) d \eta \\
& +\sum_{0<t_{k}<t} C\left(t-t_{k}\right) I_{k}\left(y\left(t_{k}\right), y^{\prime}\left(t_{k}\right)\right)+\sum_{0<t_{k}<t} S\left(t-t_{k}\right) \overline{I_{k}}\left(y\left(t_{k}\right), y^{\prime}\left(t_{k}\right)\right),
\end{aligned}
$$

and therefore

$$
\begin{aligned}
h^{\prime}(t)= & A S(t) \phi(0)+C(t)\left[x_{0}-f\left(0, \phi, x_{0}\right)\right]+f\left(t, y_{t}, y^{\prime}(t)\right) \\
& +\int_{0}^{t} A S(t-s) f\left(s, y_{s}, y^{\prime}(s)\right) d s \\
& +\int_{0}^{t} C(t-\eta) B \widetilde{W}^{-1}\left[y_{1}-C(m) \phi(0)-S(m)\left[x_{0}-f\left(0, \phi, x_{0}\right)\right]\right. \\
& \left.-\int_{0}^{m} C(m-s) f\left(s, y_{s}, y^{\prime}(s)\right) d s-\int_{0}^{t} S(m-s) v(s) d s\right](\eta) d \eta \\
& +\int_{0}^{t} C(t-s) v(s) d s+\sum_{0<t_{k}<t} A S\left(t-t_{k}\right) I_{k}\left(y\left(t_{k}\right), y^{\prime}\left(t_{k}\right)\right) \\
& +\sum_{0<t_{k}<t} C\left(t-t_{k}\right) \overline{I_{k}}\left(y\left(t_{k}\right), y^{\prime}\left(t_{k}\right)\right)
\end{aligned}
$$

where $u$ is defined as in (3.1) and $v \in S_{F, y_{t}, y^{\prime}}$.

The assumptions will give uniform estimates for $v$ and $y$ which in turn can be used to obtain the required bounds for $h$ and $h^{\prime}$ for every $y \in U_{q}$ and $h \in N_{1} y$. We have

$$
\begin{aligned}
\|h(t)\| & \leq C_{1}+C_{2} \int_{0}^{t}\left(\left\|y_{s}\right\|+\left|y^{\prime}(s)\right|\right) d s+C_{3} \int_{0}^{t} P(s) \Omega\left(\left\|y_{s}\right\|+\left|y^{\prime}(s)\right|\right) d s \\
|h|_{Z} & \leq C_{1}+C_{2} \int_{0}^{m}\left(\left\|y_{s}\right\| d s+\left|y^{\prime}(s)\right|\right)+C_{3} \int_{0}^{m} P(s) \Omega\left(\left\|y_{s}\right\|+\left|y^{\prime}(s)\right|\right) d s \\
& =l_{1} .
\end{aligned}
$$

Similarly,

$$
\begin{aligned}
\left\|h^{\prime}(t)\right\| \leq & C_{4}+C_{5}\left(\left\|y_{t}\right\|+\left\|y^{\prime}(s)\right\|\right)+C_{6} \int_{0}^{t}\left(\left\|y_{s}\right\| d s+\left\|y^{\prime}(s)\right\|\right) d s \\
& +C_{7} \int_{0}^{t} P(s) \Omega\left(\left\|y_{s}\right\|+\left\|y^{\prime}(s)\right\|\right) d s \\
\left|h^{\prime}\right|_{Z} \leq & C_{4}+C_{5}\left(\left\|y_{t}\right\|+\left\|y^{\prime}(s)\right\|\right)+C_{6} \int_{0}^{m}\left(\left\|y_{s}\right\|+\left\|y^{\prime}(s)\right\|\right) d s
\end{aligned}
$$




$$
\begin{aligned}
& +C_{7} \int_{0}^{m} P(s) \Omega\left(\left\|y_{s}\right\|+\left\|y^{\prime}(s)\right\|\right) d s \\
= & l_{2} .
\end{aligned}
$$

Step 4: $N_{1}\left(U_{q}\right)$ is equi-continuous for each $q \in \mathbb{N}$. That is, the family $\{h \in$ $\left.N_{1} y: y \in U_{q}\right\}$ is equi-continuous.

Let $U_{q}=\{y \in Z,\|y\| \leq q\}$ for some $q \geq 1$. Let $y \in U_{q}, h \in N_{1} y$ and $t_{1}, t_{2} \in J$ such that $0<t_{1}<t_{2} \leq m$. Then,

$$
\begin{aligned}
& \left|h\left(t_{1}\right)-h\left(t_{2}\right)\right| \\
& \leq\left|\left[C\left(t_{1}\right)-C\left(t_{2}\right)\right] \phi(0)\right|+\left|\left[S\left(t_{1}\right)-S\left(t_{2}\right)\right]\left[x_{0}-f\left(0, \phi, x_{0}\right)\right]\right| \\
& +\left|\int_{0}^{t_{1}}\left[C\left(t_{1}-s\right)-C\left(t_{2}-s\right)\right] f\left(s, y_{s}, y^{\prime}(s)\right) d s\right| \\
& +\left|\int_{t_{1}}^{t_{2}} C\left(t_{2}-s\right) f\left(s, y_{s}, y^{\prime}(s)\right) d s\right| \\
& +\mid \int_{0}^{t_{1}}\left[S\left(t_{1}-\eta\right)-S\left(t_{2}-\eta\right)\right] B \widetilde{W}^{-1}\left[y_{1}-C(m) \phi(0)-S(m)\left[x_{0}-f\left(0, \phi, x_{0}\right)\right]\right. \\
& -\int_{0}^{m} C(m-s) f\left(s, y_{s}, y^{\prime}(s)\right) d s-\int_{0}^{m} S(m-s) v(s) d s-\sum_{0<t_{k}<t_{1}}\left\{\left[C\left(t_{1}-t_{k}\right)\right.\right. \\
& \left.\left.-C\left(t_{2}-t_{k}\right)\right] I_{k}\left(y\left(t_{k}\right), y^{\prime}\left(t_{k}\right)\right)+\left[S\left(t_{1}-t_{k}\right)-S\left(t_{2}-t_{k}\right)\right] \overline{I_{k}}\left(y\left(t_{k}\right), y^{\prime}\left(t_{k}\right)\right)\right\} \\
& \left.-\sum_{t_{1}<t_{k}<t_{2}}\left\{C\left(t_{2}-t_{k}\right) I_{k}\left(y\left(t_{k}\right), y^{\prime}\left(t_{k}\right)\right)+S\left(t_{2}-t_{k}\right) \overline{I_{k}}\left(y\left(t_{k}\right), y^{\prime}\left(t_{k}\right)\right)\right\}\right](\eta) d \eta \\
& +\mid \int_{t_{1}}^{t_{2}} S\left(t_{2}-\eta\right) B \widetilde{W}^{-1}\left[y_{1}-C(m) \phi(0)-S(m)\left[x_{0}-f\left(0, \phi, x_{0}\right)\right]\right. \\
& -\int_{0}^{m} C(m-s) f\left(s, y_{s}, y^{\prime}(s)\right) d s-\int_{0}^{m} S(m-s) v(s) d s \\
& \left.-\sum_{t_{1}<t_{k}<t_{2}}\left\{C\left(t_{2}-t_{k}\right) I_{k}\left(y\left(t_{k}\right), y^{\prime}\left(t_{k}\right)\right)+S\left(t_{2}-t_{k}\right) \overline{I_{k}}\left(y\left(t_{k}\right), y^{\prime}\left(t_{k}\right)\right)\right\}\right](\eta) d \eta \\
& +\left|\int_{0}^{t_{1}}\left[S\left(t_{1}-s\right)-S\left(t_{2}-s\right)\right] v(s) d s\right|+\left|\int_{t_{1}}^{t_{2}} S\left(t_{2}-s\right) v(s) d s\right| \\
& +\sum_{0<t_{k}<t_{1}}\left|C\left(t_{1}-t_{k}\right) I_{k}\left(y\left(t_{k}\right), y^{\prime}\left(t_{k}\right)\right)+S\left(t_{1}-t_{k}\right) \overline{I_{k}}\left(y\left(t_{k}\right), y^{\prime}\left(t_{k}\right)\right)\right| \\
& +\sum_{t_{1}<t_{k}<t_{2}}\left|C\left(t_{2}-t_{k}\right) I_{k}\left(y\left(t_{k}\right), y^{\prime}\left(t_{k}\right)\right)+S\left(t_{2}-t_{k}\right) \overline{I_{k}}\left(y\left(t_{k}\right), y^{\prime}\left(t_{k}\right)\right)\right| .
\end{aligned}
$$

Now using the bounds on $y, v$ and the given assumptions, by a routine calculation, we obtain a positive constant $L>0$ such that

$$
\left|h\left(t_{1}\right)-h\left(t_{2}\right)\right|
$$




$$
\begin{aligned}
\leq & L\left\{\left|C\left(t_{1}\right)-C\left(t_{2}\right)\right|+\left|S\left(t_{1}\right)-S\left(t_{2}\right)\right|\right\} \\
& +L\left\{\int_{0}^{t_{1}}\left|C\left(t_{1}-s\right)-C\left(t_{2}-s\right)\right| d s+\int_{t_{1}}^{t_{2}}\left|C\left(t_{2}-s\right)\right| d s\right\} \\
& +L\left\{\int_{0}^{t_{1}}\left|S\left(t_{1}-s\right)-S\left(t_{2}-s\right)\right| d s+\int_{t_{1}}^{t_{2}}\left|S\left(t_{2}-s\right)\right| d s\right\} \\
& +L\left\{\sum_{0<t_{k}<t_{1}}\left|\left[C\left(t_{1}-t_{k}\right)+S\left(t_{1}-t_{k}\right)\right]-\left[C\left(t_{2}-t_{k}\right)-S\left(t_{2}-t_{k}\right)\right]\right|\right. \\
& \left.+\sum_{t_{1}<t_{k}<t_{2}}\left|\left[C\left(t_{2}-t_{k}\right)+S\left(t_{2}-t_{k}\right)\right]\right|\right\} .
\end{aligned}
$$

In an analogous way, one can also obtain a similar estimate for $\left|h^{\prime}\left(t_{1}\right)-h^{\prime}\left(t_{2}\right)\right|$.

Note that $C(t)$ and $S(t)$ are uniformly continuous in the uniform operator topology. Thus, the above estimates implies the required equi-continuity. This also proves the relative compactness of $N_{1}\left(U_{q}\right)$. Now it remains to prove the u.s.c of $N_{1}$. By our discussion in Section 1, it is enough to prove that $N_{1}$ has a closed graph. We do this in the next step using Lemma 2.3.

Step 5: Let $h_{n} \in N_{1} y_{n}$ and $h_{n} \longrightarrow h^{*}, y_{n} \longrightarrow y^{*}$. We must show that $h^{*} \in N_{1} y^{*}$. By definition, there exists $v_{n} \in S_{F, y_{n_{t}}, y_{n}^{\prime}}$ such that

$$
\begin{aligned}
h_{n}(t)= & C(t) \phi(0)+S(t)\left[x_{0}-f\left(0, \phi, x_{0}\right)\right]+\int_{0}^{t} C(t-s) f\left(s, y_{s}, y^{\prime}(s)\right) d s \\
& +\int_{0}^{t} S(t-s) v_{n}(s) d s+\int_{0}^{t} S(t-\eta) B u_{n}(\eta) d \eta \\
& \left.+\sum_{0<t_{k}<t}\left\{C\left(t-t_{k}\right) I_{k}\left(y_{n_{t_{k}}}, y_{n}^{\prime}\left(t_{k}\right)\right)+S\left(t-t_{k}\right) \overline{I_{k}}\left(y_{n_{t_{k}}}, y_{n}^{\prime}\left(t_{k}\right)\right)\right\}\right\},
\end{aligned}
$$

where $u_{n}$ is defined as in (3.1) and $y$ is replaced by $y_{n}$. The difficulty is that we do not have the convergence of $v_{n}$ and hence that of $u_{n}$. In fact, we cannot expect the convergence of $v_{n}$ and the existence of $v^{*}$ (to be defined later) has to be achieved by a suitable selection. First, we separate the part of $v_{n}$ from $u_{n}$. Write $u_{n}=\bar{u}_{n}+\tilde{u}_{n}$, where

$$
\begin{aligned}
\bar{u}_{n}(t)=\widetilde{W}^{-1}[ & y_{1}-C(m) \phi(0)-S(m)[x(0)-f(0, \phi)]-\int_{0}^{m} C(m-s) f\left(s, y_{n_{s}}\right) d s \\
& \left.-\sum_{0<t_{k}<t}\left\{C\left(t-t_{k}\right) I_{k}\left(y_{n_{t_{k}}}, y_{n}^{\prime}\left(t_{k}\right)\right)+S\left(t-t_{k}\right) \overline{I_{k}}\left(y_{n_{t_{k}}}, y_{n}^{\prime}\left(t_{k}\right)\right)\right\}\right](t)
\end{aligned}
$$

and

$$
\tilde{u}_{n}(t)=-\widetilde{W}^{-1}\left[\int_{0}^{m} S(m-s) v_{n}(s) d s+\sum_{0<t_{k}<t}\left\{C\left(t-t_{k}\right) I_{k}\left(y_{n_{t_{k}}}, y_{n}^{\prime}\left(t_{k}\right)\right)\right.\right.
$$




$$
\left.\left.+S\left(t-t_{k}\right) \overline{I_{k}}\left(y_{n_{t_{k}}}, y_{n}^{\prime}\left(t_{k}\right)\right)\right\}\right](t) .
$$

Thus, we get from (3.2) that

$$
\begin{aligned}
\tilde{h}_{n}(t):= & h_{n}(t)-C(t) \phi(0)-S(t)\left[x_{0}-f\left(0, \phi, x_{0}\right)\right] \\
& -\int_{0}^{t} C(t-s) f\left(s, y_{n_{s}}\right) d s-\int_{0}^{t} S(t-\eta) B \bar{u}_{n}(\eta) d \eta \\
& -\sum_{0<t_{k}<t}\left\{C\left(t-t_{k}\right) I_{k}\left(y_{n_{t_{k}}}, y_{n}^{\prime}\left(t_{k}\right)\right)+S\left(t-t_{k}\right) \overline{I_{k}}\left(y_{n_{t_{k}}}, y_{n}^{\prime}\left(t_{k}\right)\right)\right\} \\
& +\int_{0}^{t} S(t-\eta) B \tilde{u}_{n}(\eta) d \eta+\int_{0}^{t} S(t-s) v_{n}(s) d s .
\end{aligned}
$$

Note that the LHS of the above equation does not contain $v_{n}$. In order to apply Lemma 2.5, define $\Gamma: L^{1}(J, E) \rightarrow C(J, E)$ by

$$
\begin{aligned}
\Gamma(v)(t):= & -\int_{0}^{t} S(t-s) B \widetilde{W}^{-1}\left[\int_{0}^{m} S(m-\eta) v(\eta) d \eta\right](s) d s \\
& +\int_{0}^{t} S(t-s) v(s) d s .
\end{aligned}
$$

Then, $\tilde{h}_{n}(t) \in \Gamma S_{F, y_{n_{t}}, y_{n}^{\prime}}$ and since $h_{n}$ and $y_{n}$ converges, we deduce that $\tilde{h}_{n}$ also converges to $\tilde{h}^{*}$ which is given by

$$
\begin{aligned}
\tilde{h}^{*}(t):= & h^{*}(t)-C(t) \phi(0)-S(t)\left[x_{0}-f(0, \phi)\right]-\int_{0}^{t} C(t-s) f\left(s, y_{s}^{*}\right) d s \\
& -\int_{0}^{t} S(t-\eta) B \bar{u}(\eta) d \eta-\sum_{0<t_{k}<t}\left[I_{k}\left(y^{*}\left(t_{k}\right)\right)+\left(t-t_{k}\right) \overline{I_{k}}\left(y^{*}\left(t_{k}\right)\right)\right],
\end{aligned}
$$

where $\bar{u}$ has the same definition as $\bar{u}_{n}$ with $y_{n}$ replaced by $y^{*}$. Finally, from Lemma 2.5, there exists $v^{*} \in \Gamma S_{F, y_{t}^{*}, y_{*}^{\prime}}$ such that

$$
\begin{aligned}
h^{*}(t)= & C(t) \phi(0)+S(t)\left[x_{0}-f(0, \phi)\right]+\int_{0}^{t} C(t-s) f\left(s, y_{s}^{*}\right) d s \\
& +\int_{0}^{t} S(t-s) v^{*}(s) d s+\int_{0}^{t} S(t-\eta) B u^{*}(\eta) d \eta \\
& +\sum_{0<t_{k}<t}\left[I_{k}\left(y^{*}\left(t_{k}\right)\right)+\left(t-t_{k}\right) \overline{I_{k}}\left(y^{*}\left(t_{k}\right)\right)\right],
\end{aligned}
$$

where $u^{*}$ is defined as in (1.1) with $y$ replaced by $y^{*}$. Observe that we do not claim the convergence of $u_{n}$ to $u^{*}$ and $v_{n}$ to $v^{*}$.

This shows that $N_{1}$ has a closed graph. As a consequence of Lemma 2.6, we deduce that $N_{1}$ has a fixed point in $Z$. Thus, the system (1.1) is controllable on $J$ and this completes the proof of the main theorem. 


\section{Example}

Consider the following second-order partial differential inclusion:

$$
\left\{\begin{array}{l}
\left.\frac{\partial}{\partial t}\left[\frac{\partial y}{\partial t}(t, \xi)-\int_{-\infty}^{t} \int_{0}^{\pi} b(t-s, \eta, \xi) d \eta d s\right)\right] \in \frac{\partial^{2} y}{\partial \xi^{2}}(t-\xi)+u(t, \xi) \\
+\int_{-\infty}^{t} F(t, t-s, \xi, y(s, \xi)) d \xi ; t \in[0, m], \xi \in[0, \pi] \\
y(t, 0)=y(t, \pi)=0, \text { for } t \in[0, m] \\
y(\tau, \xi)=\phi(\tau, \xi), \tau \leq 0,0 \leq \xi \leq \pi \\
\frac{\partial y}{\partial t}(0, \xi)=x_{0}(y), t \in J=[0, m] \text { for } 0<y<\pi \\
\Delta y\left(t_{k}\right)(\xi)=\int_{-\infty}^{t_{k}} a_{k}\left(t_{k}-s\right) y(s, \xi) d s \\
\Delta y^{\prime}\left(t_{k}\right)(\xi)=\int_{-\infty}^{t_{k}} \tilde{a_{k}}\left(t_{k}-s\right) y(s, \xi) d s
\end{array}\right.
$$

where $0<t_{1}<t_{2}<\cdots<t_{p}<m$ and $\phi \in C_{0} \times L^{2}([0, \pi], E)$.

Let $E=L^{2}[0, \pi]$ and $C_{r}=C([-r, 0] ; E)$ be as in Section 1 .

(a) The functions $b(s, \eta, \xi), \frac{\partial b}{\partial \xi}(s, \eta, \xi)$ are completely continuous and satisfy the growth conditions.

(b) The function $F: \mathbb{R}^{4} \rightarrow \mathbb{R}$ is Caratheodory and there exist a measurable function $\nu: \mathbb{R}^{3} \rightarrow \mathbb{R}$ and an upper semi-continuous function $\zeta: \mathbb{R}^{3} \rightarrow \mathbb{R}$ such that

$$
\|F(t, s, \xi, x)\| \leq \nu(t, s, \xi)+\zeta(t, s)\|x\|,(t, s, \xi, x) \in \mathbb{R}^{4}, t \in[0, m] .
$$

Moreover, $f(t, \cdot), I_{k} ; k=1,2, \ldots, p$, are bounded linear operators, and

$$
\left\|I_{k}\right\| \leq c_{k},\left\|\overline{I_{k}}\right\| \leq d_{k} ; k=1,2, \ldots, p .
$$

(c) The functions $a_{k}, \tilde{a_{k}} \in C([0, m], \mathbb{R})$ for $k=1,2, \ldots, p$.

Assume that conditions (a)-(c) are satisfied. The problem can be modelled as the abstract impulsive problem (1.1) by defining

$$
\begin{gathered}
f(t, \psi)(\xi):=\int_{-\infty}^{0} \int_{0}^{\pi} b(s, \nu, \xi) \psi(s, \nu) d \nu d s \\
F(t, \psi, x)(\xi):=\int_{-\infty}^{0} F(t, s, \xi, \psi(s, \xi)) d s \\
I_{k}(\psi)(\xi)=\int_{-\infty}^{0} a_{k}(s) \psi(s, \xi) d s, \overline{I_{k}}(\psi)(\xi)=\int_{-\infty}^{0} \tilde{a_{k}}(s) \psi(s, \xi) d s .
\end{gathered}
$$

Here, $u:(0, \pi) \times J \rightarrow \mathbb{R}$ is continuous in $t$ which is the control function.

Define $A: E \rightarrow E$ by $A \omega=\omega^{\prime \prime} ; \omega \in D(A)$, where

$D(A)=\left\{\omega \in E: \omega, \omega^{\prime}\right.$ are absolutely continuous, $\left.\omega^{\prime \prime} \in E, \omega(0)=\omega(\pi)=0\right\}$.

Then A has a spectral representation

$$
A \omega=\Sigma_{n=1}^{\infty}-n^{2}\left(\omega, \omega_{n}\right) \omega_{n}, \omega \in D(A),
$$


where $\omega_{n}(s)=\sqrt{\frac{2}{\pi}} \sin n s ; n=1,2,3, \ldots$ is the orthogonal set of eigen-functions of $A$. Further, it can be shown that $A$ is the infinitesimal generator of a strongly continuous Cosine family $C(t), t \in \mathbb{R}$ defined on $E$ which is given by

$$
C(t) \omega=\Sigma_{n=1}^{\infty} \cos n t\left(\omega, \omega_{n}\right) \omega_{n}, \omega \in E .
$$

The associated Sine family is given by

$$
S(t) \omega=\Sigma_{n=1}^{\infty} \frac{1}{n} \sin n t\left(\omega, \omega_{n}\right) \omega_{n}, \omega \in E .
$$

The control operator $B: L^{2}(J, E) \rightarrow E$ is defined by

$$
(B u)(t)(\xi)=u(t, \xi)
$$

which satisfies the condition (H2). Now the PDE (4.1) can be represented in the form (1.1). Hence, by Section 3 the system is controllable.

In particular, if $F\left(t, y_{t}, y^{\prime}(t)\right)$ is a single valued map, then the system (1.1) becomes

$$
\left\{\begin{array}{l}
\frac{d}{d t}\left[y^{\prime}(t)-f\left(t, y_{t}, y^{\prime}(t)\right)\right]=A y(t)+B u(t)+F\left(t, y_{t}, y^{\prime}(t)\right) ; \\
t \in J:=[0, \infty) ; t \neq t_{k} ; \\
\left.\Delta y\right|_{t=t_{k}}=I_{k}\left(y_{t_{k}}, y^{\prime}\left(t_{k}\right)\right) ; k=1,2, \ldots, p, \\
\left.\Delta y^{\prime}\right|_{t=t_{k}}=\overline{I_{k}}\left(y_{t_{k}}, y^{\prime}\left(t_{k}\right)\right) ; k=1,2, \ldots, p, \\
y_{0}=\phi ; y^{\prime}(0)=x_{0} .
\end{array}\right.
$$

By using Sadovskii's fixed-point theorem, we can analogously study the controllability of the system (4.2).

Hypothesis(H41) The function $\mathrm{F}: J \times \mathcal{B}_{h} \times E \rightarrow E$ satisfies the following condition, for each $t \in J$, the function $F(t, \cdot, \cdot): \mathcal{B}_{h} \times E \rightarrow E$ is continuous; and for each $\psi \in \mathcal{B}_{h}, y \in E$, the function $F(\cdot, \psi, y): J \rightarrow E$ is strongly measurable.

Theorem 4.1. If the hypotheses (H1)-(H3), (H41), (H5), and (H6) are satisfied, then the system (4.2) is controllable on $J$.

Proof. The mild solution of the system (4.2) is given by

$$
\begin{aligned}
y(t)= & C(t) \phi(0)+S(t)\left[x_{0}-f\left(0, \phi, x_{0}\right)\right]+\int_{0}^{t} C(t-s) f\left(s, y_{s}, y^{\prime}(s)\right) d s \\
& +\int_{0}^{t} S(t-s) B u(s) d s+\int_{0}^{t} S(t-s) F\left(s, y_{s}, y^{\prime}(s)\right) d s \\
& +\sum_{0<t_{k}<t} C\left(t-t_{k}\right) I_{k}\left(y_{t_{k}}, y^{\prime}\left(t_{k}\right)\right)+\sum_{0<t_{k}<t} S\left(t-t_{k}\right) \overline{I_{k}}\left(y_{t_{k}}, y^{\prime}\left(t_{k}\right)\right) .
\end{aligned}
$$

We define an operator $N: Z \rightarrow Z$ by

$$
\begin{aligned}
(N y)(t)= & C(t) \phi(0)+S(t)\left[x_{0}-f\left(0, \phi, x_{0}\right)\right]+\int_{0}^{t} C(t-s) f\left(s, y_{s}, y^{\prime}(s)\right) d s \\
& +\int_{0}^{t} S(t-s) B u(s) d s+\int_{0}^{t} S(t-s) F\left(s, y_{s}, y^{\prime}(s)\right) d s
\end{aligned}
$$




$$
+\sum_{0<t_{k}<t} C\left(t-t_{k}\right) I_{k}\left(y_{t_{k}}, y^{\prime}\left(t_{k}\right)\right)+\sum_{0<t_{k}<t} S\left(t-t_{k}\right) \bar{I}_{k}\left(y_{t_{k}}, y^{\prime}\left(t_{k}\right)\right) .
$$

Then we can decompose $N$ into $N=N_{1}+N_{2}$, where

$$
\begin{aligned}
\left(N_{1} y\right)(t)= & \int_{0}^{t} C(t-s) f\left(s, y_{s}, y^{\prime}(s)\right) d s+\int_{0}^{t} S(t-s) B u(s) d s \\
& +\int_{0}^{t} S(t-s) F\left(s, y_{s}, y^{\prime}(s)\right) d s
\end{aligned}
$$

and

$$
\begin{aligned}
\left(N_{2} y\right)(t)= & C(t) \phi(0)+S(t)\left[x_{0}-f\left(0, \phi, x_{0}\right)\right]+\sum_{0<t_{k}<t} C\left(t-t_{k}\right) I_{k}\left(y_{t_{k}}, y^{\prime}\left(t_{k}\right)\right) \\
& +\sum_{0<t_{k}<t} S\left(t-t_{k}\right) \bar{I}_{k}\left(y_{t_{k}}, y^{\prime}\left(t_{k}\right)\right),
\end{aligned}
$$

and verify that $N_{1}$ is a contraction while $N_{2}$ is a compact operator. Thus, by Sadovskii's fixed- point theorem, the operator $N$ has at least one fixed point on $Z$, which shows the controllability of the system (4.2) on interval $J$. The proof is similar to Step 4 of Theorem 3.1.

\section{Exact controllability in infinite dimensional space}

In Section 4, we have proved controllability result with compactness of semigroup/ Cosine operator. But if the semigroup is compact then the assumption (H2) in Section 2 and assumption (H2) in [19] are valid only in finite dimensional space so the applications are restricted to ordinary differential control systems but not to partial differential equations. Thus example given in Section 4 and $([2],[4],[5],[19])$ cannot be recovered as of the abstract result in infinite dimension.

Lemma 5.1. Let $\mathcal{P C}([0, \tau]) ; E)$ be a space formed by normalized piecewise continuous functions on $([0, \tau] ; E)$. Let $\mathcal{B}_{h} \subseteq \mathcal{P C}$ such that $\widetilde{\mathcal{B}}_{k}=\left\{\widetilde{V_{k}}: V \in \mathcal{B}_{h}\right\}$, where

$$
\widetilde{V_{k}}(t)=\left\{\begin{array}{l}
V(t) ; t \in\left(t_{k}, t_{k+1}\right], \\
V\left(t_{k}^{+}\right) ; t=t_{k} .
\end{array}\right.
$$

The set $\mathcal{B}_{h} \subseteq \mathcal{P C}$ is relatively compact if and only if each set $\widetilde{\mathcal{B}}_{k}$ is relatively compact in the space $C\left(\left[t_{k}, t_{k+1}\right] ; E\right)$.

Proof. For the proof refer to [14].

Hypothesis(H11) $A: D(A) \rightarrow E$ is the infinitesimal generator of an uniformly bounded analytic Cosine operators $C(t) ; t \in \mathbb{R}$ on $E$. We define the fractional power $(-A)^{\alpha}, 0<\alpha \leq 1$, as a closed linear operator on its domain $D\left((-A)^{\alpha}\right)$ which is dense in $E$ and the expression $\|y\|_{\alpha}=\left\|(-A)^{\alpha} y\right\|$ is the norm in $D\left((-A)^{\alpha}\right)$. 
One has to be precise for the meaning of derivative in system (4.2). $x \in \mathcal{P C}$ is piecewise smooth if $x$ is continuously differentiable at $t \neq t_{i}, i=0,1,2, \ldots, n+1$; and for $t=t_{i}, i=0,1,2, \ldots, n$, there exists right side derivative $x_{R}^{\prime}(t)=$ $\lim _{s \rightarrow 0^{+}} \frac{x(t+s)-x\left(t^{+}\right)}{s}$, while for $t=t_{i}, i=1,2, \ldots, n+1$, there exists left side derivative $x_{L}^{\prime}(t)=\lim _{s \rightarrow 0^{-}} \frac{x(t+s)-x\left(t^{-}\right)}{s}$. Also, $x^{\prime}(0)$ corresponds to $x_{R}^{\prime}(0)$ and we represent by $x^{\prime}(t)$, the left derivative of $x$ at $t>0$. Furthermore, we denote by $\mathcal{P C}^{1}$ the space of piecewise smooth functions (in above sense) endowed with the norm $|u|_{1}=|u|+\left|u^{\prime}\right|$.

Lemma 5.2. Let $h_{1}:[0, m] \rightarrow E_{1}$ be an integrable function such that $h_{1} \in \mathcal{P C}$. Then

(i) the function $v(t)=\int_{0}^{t} C(t-s) h_{1}(s) d s \in \mathcal{P C}^{1}$,

(ii) the function $s \rightarrow A S(t-s) h_{1}(s)$ is integrable on $[0, t], t \in[0, m]$, and

(iii) $v^{\prime}(t)=h_{1}(t)+A \int_{0}^{t} S(t-s) h_{1}(s) d s, t \in J$.

Proof. For the proof refer to [15].

We assume following hypotheses.

Hypothesis(H31) There exists $0<\beta<1$ such that $f(\cdot)$ is $E_{\beta}$-valued and

(i) For every $y:(-\infty, m] \rightarrow E$ such that $y_{0} \in \mathcal{B}_{h}$ and $\left.y\right|_{J} \in \mathcal{P C}$, the function $t \rightarrow(-A)^{\beta} f\left(t, y_{t}, y^{\prime}(t)\right)$ is continuous on $J$.

(ii) For each $t \in J$, the function $(-A)^{\beta} f(t, \cdot, \cdot): \mathcal{B}_{h} \times E \rightarrow E$ is continuous and there exist positive constants $c_{1}$ and $c_{2}$ such that

$$
\left\|(-A)^{\beta} f(t, \phi, y)\right\| \leq c_{1}\left(\|\phi\|_{\mathcal{B}_{h}}+|y|\right)+c_{2},(t, \phi, y) \in J \times \mathcal{B}_{h} \times E .
$$

Hypothesis(H42) The function $F: J \times \mathcal{B}_{h} \times E \rightarrow E$ satisfies,

(i) For every $y:(-\infty, m] \rightarrow E$ such that $y_{0}=\phi$ and $\left.y\right|_{J} \in \mathcal{P C} \mathcal{C}^{1}$, the function $t \rightarrow F\left(t, y_{t}, y^{\prime}(t)\right)$ is strongly measurable.

(ii) For each $t \in J$, the function $F(t, \cdot, \cdot): \mathcal{B}_{h} \times E \rightarrow E$ is continuous.

(iii) There exists an integrable function $P_{F}: J \rightarrow[0, \infty)$ and a continuous nondecreasing function $\Omega:[0, \infty) \rightarrow(0, \infty)$ such that

$$
\|F(t, \phi, y)\| \leq P(t) \Omega\left(\|\phi\|_{\mathcal{B}_{h}}+|y|\right),(t, \phi, y) \in J \times \mathcal{B}_{h} \times E .
$$

(H7) There exist continuous functions $f_{1}: J \times E_{1} \rightarrow E_{1}$ and $f_{2}: J \times \mathcal{B}_{h} \rightarrow E_{1}$ such that $f(t, \phi, y)=f_{1}(t, y)+f_{2}(t, \phi)$ and the following conditions are fulfilled:

(i) The function $f_{2}: J \times \mathcal{B}_{h} \rightarrow E_{1}$ is completely continuous and there exists a continuous nondecreasing function $\Omega:[0, \infty) \rightarrow(0, \infty)$ and $L_{f}>0$ such that

$$
\begin{gathered}
\left|f_{1}(t, x)-f_{1}(t, y)\right| \leq L_{f}|x-y| ; t \in J ; x, y \in E . \\
\left|f_{2}(t, \phi)\right|_{E_{1}} \leq \Omega\left(\|\phi\|_{\mathcal{B}_{h}}\right) ;(t, \phi) \in J \times \mathcal{B}_{h} .
\end{gathered}
$$

(ii) Let $S(m)=\left\{y:(-\infty, m] \rightarrow E: y_{0}=0,\left.y\right|_{J} \in \mathcal{P C}\right\}$ endowed with the norm of uniformly convergence. The functions $v_{y}(t)=f_{1}(t, y(t))$ and $w_{y}(t)=$ $f_{2}\left(t, y_{t}\right)$ are continuous on $J$, and the set of functions $\left\{\widetilde{\left(w_{y}\right)}:: y \in \mathcal{P C}, \mid y \|_{J} \leq r\right\}$ is uniformly equi-continuous on $\left[t_{i}, t_{i+1}\right] ; i=0,1,2, \ldots, p$. 
Theorem 5.3. Assume that $F$ verifies (H42) and $g$ verifies (H31). Suppose that $\phi(0) \in E_{1}$ and following conditions are fulfilled:

(a) For every bounded set $B_{1} \subseteq \mathcal{B}_{h} \times E$, the set $F\left(J \times B_{1}\right)$ is relatively compact in $E$.

(b) Assumptions (H11), (H2), (H5), (H7) hold, and

$$
\begin{aligned}
& L_{f}\left\{m\left(M+N_{1}\right)+1\right\}+K_{m}\left\{\left(m+N_{1}\right) \sum_{k=1}^{p} c_{k}^{1}+(M m+N) \sum_{k=1}^{p} d_{k}^{1}\right\} \\
& +K_{m} \lim _{\xi \rightarrow+\infty} \inf \left\{M m \frac{\Omega_{F}(\xi)}{\xi} \int_{0}^{m} P(s) d s+\frac{\Omega_{F}(\xi)}{\xi}\right. \\
& \left.+M \frac{\Omega_{F}(\xi)}{\xi} \int_{0}^{m} P(s) d s+M m \frac{\Omega_{F}(\xi)}{\xi}+N_{1} m \frac{\Omega_{F}(\xi)}{\xi}\right\} \\
& +\frac{M M_{2}}{r}\left\{(m+1) \int_{0}^{t}\left|u_{1}^{r}(s)\right| d s+m M_{3} \int_{0}^{t}\left|u_{2}^{r}(s)\right| d s\right\}<1 .
\end{aligned}
$$

Then the system (4.2) is controllable on J.

Proof. Let

$$
S^{1}(m)=\left\{S(m)=y:(-\infty, m] \rightarrow E: y_{0}=0,\left.y\right|_{J} \in \mathcal{P} \mathcal{C}^{1}, y^{\prime}(0)=-f\left(0, \phi, x_{0}\right)\right\}
$$

endowed with the norm of $\mathcal{P C}^{1}$. Clearly, $S^{1}(m)$ is a closed and convex subset of $\mathcal{P} \mathcal{C}^{1}$. Define the operator $\Gamma: S^{1}(m) \rightarrow S^{1}(m)$ by

$$
\begin{aligned}
(\Gamma z)(t)= & \int_{0}^{t} C(t-s) f_{1}\left(s, z^{\prime}(s)+x^{\prime}(s)\right) d s+\int_{0}^{t} C(t-s) f_{2}\left(s, z_{s}+x_{s}\right) d s \\
& +\int_{0}^{t} S(t-s) F\left(s, z_{s}+x_{s}, z^{\prime}(s)+x^{\prime}(s)\right) d s \\
& +\sum_{0<t_{k}<t} C\left(t-t_{k}\right) I_{k}\left(z_{t_{k}}+x_{t_{k}}, z^{\prime}\left(t_{k}\right)+x^{\prime}\left(t_{k}\right)\right) \\
& +\sum_{0<t_{k}<t}^{t} S\left(t-t_{k}\right) \overline{I_{k}}\left(z_{t_{k}}+x_{t_{k}}, z^{\prime}\left(t_{k}\right)+x^{\prime}\left(t_{k}\right)\right) \\
& +\int_{0}^{t} S(t-s) B \widetilde{W}^{-1}\left\{-\int_{0}^{m} C(m-\eta) f_{1}\left(\eta, z^{\prime}(\eta)+x^{\prime}(\eta)\right) d \eta\right. \\
& -\int_{0}^{m} C(m-\eta) f_{2}\left(\eta, z_{\eta}+x_{\eta}\right) d \eta \\
& -\int_{0}^{m} S(m-\eta) F\left(\eta, z_{\eta}+x_{\eta}, z^{\prime}(\eta)+x^{\prime}(\eta)\right) d \eta \\
& -\sum_{0<t_{k}<t} C\left(t-t_{k}\right) I_{k}\left(z_{t_{k}}+x_{t_{k}}, z^{\prime}\left(t_{k}\right)+x^{\prime}\left(t_{k}\right)\right)
\end{aligned}
$$




$$
\left.-\sum_{0<t_{k}<t} S\left(t-t_{k}\right) \overline{I_{k}}\left(z_{t_{k}}+x_{t_{k}}, z^{\prime}\left(t_{k}\right)+x^{\prime}\left(t_{k}\right)\right)\right\} d s ; t \in J .
$$

$(\Gamma z)^{\prime}(t)$

$$
\begin{aligned}
= & f_{1}\left(t, z^{\prime}(t)+x^{\prime}(t)\right)+f_{2}\left(t, z_{t}+x_{t}\right)+\int_{0}^{t} A S(t-s) f_{1}\left(s, z^{\prime}(s)+x^{\prime}(s)\right) d s \\
& +\int_{0}^{t} A S(t-s) f_{2}\left(s, z_{s}+x_{s}\right) d s+\int_{0}^{t} C(t-s) F\left(s, z_{s}+x_{s}, z^{\prime}(s)+x^{\prime}(s)\right) d s \\
& +\sum_{0<t_{k}<t} A S\left(t-t_{k}\right) I_{k}\left(z_{t_{k}}+x_{t_{k}}, z^{\prime}\left(t_{k}\right)+x^{\prime}\left(t_{k}\right)\right) \\
& +\sum_{0<t_{k}<t} C\left(t-t_{k}\right) \bar{I}_{k}\left(z_{t_{k}}+x_{t_{k}}, z^{\prime}\left(t_{k}\right)+x^{\prime}\left(t_{k}\right)\right) \\
& +\int_{0}^{t} C(t-s) B u(s) d s \\
& +\int_{0}^{t} S(t-s) B \widetilde{W}-1\left\{-\int_{0}^{m} A S(m-\eta) f_{1}\left(\eta, z^{\prime}(\eta)+x^{\prime}(\eta)\right) d \eta\right. \\
& -\int_{0}^{m} A S(m-\eta) f_{2}\left(\eta, z_{\eta}+x_{\eta}\right) d \eta \\
& -\int_{0}^{m} C(m-\eta) F\left(\eta, z_{\eta}+x_{\eta}, z^{\prime}(\eta)+x^{\prime}(\eta)\right) d \eta \\
& \left.-\sum_{0<t_{k}<t} A S\left(t-t_{k}\right) I_{k}\left(z_{t_{k}}+x_{t_{k}}, z^{\prime}\left(t_{k}\right)+x^{\prime}\left(t_{k}\right)\right)\right\} d s ; t \in J .
\end{aligned}
$$

Clearly $\Gamma$ is continuous using (H31) and (H42). Next we prove that there exists $r_{0} \geq 0$ such that $\Gamma\left(B_{r_{0}}\left(0, S^{1}(m)\right)\right) \subset B_{r_{0}}\left(0, S^{1}(m)\right)$. Assume that this is false; then for every $r>0$ (which is different ' $r$ ' then Section 3 ), there exists $z^{r} \in B\left(0, S^{1}(m)\right)$ such that $\left|\Gamma z^{r}\right|_{1}>r$. So we have

$$
\begin{aligned}
r \leq & \left|\Gamma z^{r}+\left(\Gamma\left(z^{r}\right)\right)^{\prime}\right|_{1} \leq\left|\Gamma z^{r}\right|+\left|\left(\Gamma\left(z^{r}\right)\right)^{\prime}\right| \\
\leq & M \int_{0}^{t}\left|f_{1}\left(s,\left(z^{r}\right)^{\prime}(s)+x^{\prime}(s)\right)\right| d s+M \int_{0}^{t}\left|f_{2}\left(s, z_{s}^{r}+x_{s}\right)\right| d s \\
& +M m \int_{0}^{t}\left|F\left(s, z_{s}^{r}+x_{s},\left(z^{r}\right)^{\prime}(s)+x^{\prime}(s)\right)\right| d s \\
& +M \sum_{0<t_{k}<t}\left|I_{k}\left(z_{t_{k}}^{r}+x_{t_{k}},\left(z^{r}\right)^{\prime}\left(t_{k}\right)+x^{\prime}\left(t_{k}\right)\right)\right| \\
& +M m \sum_{0<t_{k}<t}\left|\overline{I_{k}}\left(z_{t_{k}}^{r}+x_{t_{k}},\left(z^{r}\right)^{\prime}\left(t_{k}\right)+x^{\prime}\left(t_{k}\right)\right)\right|
\end{aligned}
$$


CONTROLLABILITY OF SECOND ORDER SEMI-LINEAR NEUTRAL IMPULSIVE 835

$$
\begin{aligned}
& +M m M_{2} \int_{0}^{t}\left[M \int_{0}^{m}\left|f_{1}\left(\eta,\left(z^{r}\right)^{\prime}(\eta)+x^{\prime}(\eta)\right)\right| d \eta\right. \\
& +M \int_{0}^{m}\left|f_{2}\left(\eta, z_{\eta}^{r}+x_{\eta}\right)\right| d \eta+M m \int_{0}^{m}\left|F\left(\eta, z_{\eta}^{r}+x_{\eta},\left(z^{r}\right)^{\prime}(\eta)+x^{\prime}(\eta)\right)\right| d \eta \\
& +M \sum_{0<t_{k}<t}\left|I_{k}\left(z_{t_{k}}^{r}+x_{t_{k}},\left(z^{r}\right)^{\prime}\left(t_{k}\right)+x^{\prime}\left(t_{k}\right)\right)\right| \\
& \left.+M m \sum_{0<t_{k}<t}\left|\overline{I_{k}}\left(z_{t_{k}}^{r}+x_{t_{k}},\left(z^{r}\right)^{\prime}\left(t_{k}\right)+x^{\prime}\left(t_{k}\right)\right)\right|\right] d s \\
& +\left|f_{1}\left(t,\left(z^{r}\right)^{\prime}(t)+x^{\prime}(t)\right)\right|+\left|f_{2}\left(t, z_{t}^{r}+x_{t}\right)\right| \\
& +\int_{0}^{t}\left|A S(t-s) \| f_{1}\left(s,\left(z^{r}\right)^{\prime}(s)+x^{\prime}(s)\right)\right| d s \\
& +\int_{0}^{t}|A S(t-s)|\left|f_{2}\left(s, z_{s}^{r}+x_{s}\right)\right| d s+M \int_{0}^{t}\left|F\left(s, z_{s}^{r}+x_{s},\left(z^{r}\right)^{\prime}(s)+x^{\prime}(s)\right)\right| d s \\
& +\sum_{0<t_{k}<t}\left|A S\left(t-t_{k}\right)\right|\left|I_{k}\left(z_{t_{k}}^{r}+x_{t_{k}},\left(z^{r}\right)^{\prime}\left(t_{k}\right)+x^{\prime}\left(t_{k}\right)\right)\right| \\
& +M \sum_{0<t_{k}<t}\left|\overline{I_{k}}\left(z_{t_{k}}^{r}+x_{t_{k}},\left(z^{r}\right)^{\prime}\left(t_{k}\right)+x^{\prime}\left(t_{k}\right)\right)\right|+M M_{2} \int_{0}^{t}\left|u_{1}^{r}(s)\right| d s \\
& +M m M_{2} M_{3} \int_{0}^{t}\left|u_{2}^{r}(s)\right| d s \\
& \leq M \int_{0}^{m} L_{f}\left(r+\left|x^{\prime}\right|_{m}+\left|f_{1}(s, 0)\right|\right) d s+m M\left(K_{m} r+\sup _{s \in J}\left\|x_{s}\right\|_{\mathcal{B}_{h}}\right) \\
& +M m \int_{0}^{m} P(s) \Omega_{F}\left(K_{m} r+\sup _{s \in J}\left(\left\|x_{s}\right\|_{\mathcal{B}_{h}}+\left|x^{\prime}\right|_{m}\right)\right) d s \\
& +M \sum_{k=1}^{p} c_{k}^{1}\left(K_{m} r+\left\|x_{t_{k}}\right\|_{\mathcal{B}_{h}}+\left|x^{\prime}\left(t_{k}\right)\right|\right) \\
& +c_{k}^{2}+M m \sum_{k=1}^{p} d_{k}^{1}\left(K_{m} r+\left\|x_{t_{k}}\right\|_{\mathcal{B}_{h}}+\left|x^{\prime}\left(t_{k}\right)\right|\right)+d_{k}^{2}+M m M_{2} \int_{0}^{t}\left|u_{1}^{r}(s)\right| d s \\
& +L_{f}\left(r+\left|x^{\prime}\right|_{m}\right)+\left|f_{1}(t, 0)\right|+\Omega_{F}\left(K_{m} r+\sup _{s \in J}\left\|x_{t}\right\|\right) \\
& +N_{1} \int_{0}^{m} L_{f}\left(r+\left|x^{\prime}\right|_{m}+\left|f_{1}(s, 0)\right|_{1}\right) d s+N_{1} m \Omega_{F}\left(K_{m} r+\sup _{s \in J}\left\|x_{s}\right\|_{\mathcal{B}_{h}}\right) \\
& +M \int_{0}^{t} P(s) \Omega_{F}\left(K_{m} r+\sup _{s \in J}\left(\left\|x_{s}\right\|_{\mathcal{B}_{h}}+\left|x^{\prime}\right|_{m}\right)\right) d s \\
& +N_{1} \sum_{k=1}^{p} c_{k}^{1}\left(K_{m} r+\left\|x_{t_{k}}\right\|_{\mathcal{B}_{h}}+\left|x^{\prime}\left(t_{k}\right)\right|\right)+c_{k}^{2}
\end{aligned}
$$




$$
\begin{aligned}
+ & M \sum_{k=1}^{p} d_{k}^{1}\left(K_{m} r+\left\|x_{t_{k}}\right\|_{\mathcal{B}_{h}}+\left|x^{\prime}\left(t_{k}\right)\right|\right)+d_{k}^{2} \\
+ & M M_{2} \int_{0}^{t}\left|u_{1}^{r}(s)\right| d s+M m M_{2} M_{3} \int_{0}^{t}\left|u_{2}^{r}(s)\right| d s, \\
r \leq & {\left[L_{f}\left\{m\left(M+N_{1}\right)+1\right\}+K_{m}\left\{\left(m+N_{1}\right) \sum_{k=1}^{p} c_{k}^{1}+(M m+N) \sum_{k=1}^{p} d_{k}^{1}\right\}\right.} \\
& +K_{m} \lim _{\xi \rightarrow+\infty} \inf \left\{M m \frac{\Omega_{F}(\xi)}{\xi} \int_{0}^{m} P(s) d s+\frac{\Omega_{F}(\xi)}{\xi}\right. \\
& \left.+M \frac{\Omega_{F}(\xi)}{\xi} \int_{0}^{m} P(s) d s+M m \frac{\Omega_{F}(\xi)}{\xi}+N_{1} m \frac{\Omega_{F}(\xi)}{\xi}\right\} \\
& \left.+\frac{M M_{2}}{r}\left\{(m+1) \int_{0}^{t}\left|u_{1}^{r}(s)\right| d s+m M_{3} \int_{0}^{t}\left|u_{2}^{r}(s)\right| d s\right\}\right]
\end{aligned}
$$

which yields that

$$
\begin{aligned}
& L_{f}\left\{m\left(M+N_{1}\right)+1\right\}+K_{m}\left\{\left(m+N_{1}\right) \sum_{k=1}^{p} c_{k}^{1}+(M m+N) \sum_{k=1}^{p} d_{k}^{1}\right\} \\
& +K_{m} \lim _{\xi \rightarrow+\infty} \inf \left\{M m \frac{\Omega_{F}(\xi)}{\xi} \int_{0}^{m} P(s) d s+\frac{\Omega_{F}(\xi)}{\xi}\right. \\
& \left.+M \frac{\Omega_{F}(\xi)}{\xi} \int_{0}^{m} P(s) d s+M m \frac{\Omega_{F}(\xi)}{\xi}+N_{1} m \frac{\Omega_{F}(\xi)}{\xi}\right\} \\
& +\frac{M M_{2}}{r}\left\{(m+1) \int_{0}^{t}\left|u_{1}^{r}(s)\right| d s+m M_{3} \int_{0}^{t}\left|u_{2}^{r}(s)\right| d s\right\} \geq 1 .
\end{aligned}
$$

This contradicts the equation (5.1).

Now we consider the decomposition $\Gamma=\Gamma_{1}+\Gamma_{2}$, where

$$
\begin{aligned}
\left(\Gamma_{2} z\right)(t)= & \int_{0}^{t} C(t-s) f_{2}\left(s, z_{s}+x_{s}\right) d s \\
& +\int_{0}^{t} S(t-s) F\left(s, z_{s}+x_{s}, z^{\prime}(s)+x^{\prime}(s)\right) d s \\
& +\sum_{0<t_{k}<t} C\left(t-t_{k}\right) I_{k}\left(z_{t_{k}}+x_{t_{k}}, z^{\prime}\left(t_{k}\right)+x^{\prime}\left(t_{k}\right)\right) \\
& +\sum_{0<t_{k}<t} S\left(t-t_{k}\right) \overline{I_{k}}\left(z_{t_{k}}+x_{t_{k}}, z^{\prime}\left(t_{k}\right)\right. \\
& \left.+x^{\prime}\left(t_{k}\right)\right)+\int_{0}^{t} S(t-s) B \widetilde{W}^{-1}\left\{-\int_{0}^{m} C(m-\eta) f_{2}\left(\eta, z_{\eta}+x_{\eta}\right) d \eta\right. \\
& -\int_{0}^{m} S(m-\eta) F\left(\eta, z_{\eta}+x_{\eta}, z^{\prime}(\eta)+x^{\prime}(\eta)\right) d \eta
\end{aligned}
$$




$$
\begin{aligned}
& -\sum_{0<t_{k}<t} C\left(t-t_{k}\right) I_{k}\left(z_{t_{k}}+x_{t_{k}}, z^{\prime}\left(t_{k}\right)+x^{\prime}\left(t_{k}\right)\right) \\
& \left.-\sum_{0<t_{k}<t} S\left(t-t_{k}\right) \overline{I_{k}}\left(z_{t_{k}}+x_{t_{k}}, z^{\prime}\left(t_{k}\right)+x^{\prime}\left(t_{k}\right)\right)\right\} d s .
\end{aligned}
$$

Using the axiom of the phase space $\mathcal{B}_{h}$, it is easy to observe that

$$
\left\|\Gamma_{1} z_{1}-\Gamma_{1} z_{2}\right\| \leq L_{f}\left(m\left(M+N_{1}\right)+1\right)\left\|z_{1}-z_{2}\right\|_{1} ; z_{1}, z_{2} \in S^{1}(m) .
$$

This implies that $\Gamma_{1}$ is a contradiction on $B_{r_{0}}\left(0, S^{1}(m)\right)$. Moreover, the proof of Theorem 5.2 of [8] helps us to show that $\Gamma_{2}: S^{1}(m) \rightarrow \mathcal{P C}$ is completely continuous. On the other hand, using the compactness properties of the functions $F, f_{2}, I_{k}^{j}, j=1,2$ and proceeding in similar way we can show that $\left(\Gamma_{2} z\right)^{\prime}: B_{r_{0}}\left(0, S^{1}(m)\right) \rightarrow \mathcal{P C}$ is completely continuous. This proves that $\left(\Gamma_{2} z\right)^{\prime}: B_{r_{0}}\left(0, S^{1}(m)\right) \rightarrow \mathcal{P C}$ is completely continuous. Hence the proof is complete.

Acknowledgement. Authors express their gratitude to the anonymous referee for valuable comments and suggestions which are helpful to modify the manuscript.

\section{References}

[1] J. Banas and K. Goebel, Measures of Noncompactness in Banach Spaces, MarcelDekker, New York, 1980.

[2] M. Benchohra and S. K. Ntouyas, Controllability for functional differential and integrodifferential inclusions in Banach spaces, J. Optim. Theory Appl. 113 (2002), no. 3, 449-472.

[3] — Controllability results for multivalued semilinear neutral functional equations, Math. Sci. Res. J. 6 (2002), no. 2, 65-77.

[4] _ Existence and controllability results for nonlinear differential inclusions with nonlocal conditions in Banach spaces, J. Appl. Anal. 8 (2002), no. 1, 33-48.

[5] - On second order impulsive functional differential equations in Banach spaces, J. Appl. Math. Stoch. Anal. 15 (2002), no. 1, 47-55.

[6] J. Bochenek, An abstract nonlinear second-order differential equation, Ann. Polon. Math. 54 (1991), no. 2, 155-166.

[7] D. N. Chalishajar, Controllability of nonlinear integro-differential third order dispersion system, J. Math. Anal. Appl. 348 (2008), no. 1, 480-486.

[8] D. N. Chalishajar and F. S. Acharya, Controllability of nonlinear integro-differential third order dispersion inclusions in Banach spaces, Nonl. Anal. TMA, Communicated.

[9] D. N. Chalishajar, R. K. George, and A. K. Nandakumaran, Exact controllability of the nonlinear third-order dispersion equation, J. Math. Anal. Appl. 332 (2007), no. 2, 1028-1044.

[10] K. Deimling, Multivalued Differential Equations, Walter de Gruyter, Berlin, 1992.

[11] J. Dugunji and A. Granas, Fixed Point Theory. I, Monografic. Math. PWN Warsaw, 1982.

[12] R. K. George, D. N. Chalishajar, and A. K. Nandakumaran, Contollability of second order semi-linear neutral functional differential inclusions in Banach spaces, Mediterr. J. Math. 99 (2004), 1-16. 
[13] J. A. Goldstein, Semigroups of Linear Operators and Applications, Oxford Uni. Press, New-York, 1985.

[14] A. Granas and J. Dugunji, Fixed Point Theory, Springer-Verlag, New-York, 2003.

[15] E. M. Hernandez, M. Rabello, and H. Henriquez, Existence of solutions of impulsive partial neutral functional differential equations, J. Math. Anal. Appl. 331 (2007), 11351158.

[16] Sh. Hu and N. S. Papageogiou, Handbook of Multivalued Analysis, Kluwer Dordrecht, 1997.

[17] J. Kisynski, On second order Cauchy problem in a Banach space, Bull. Acad. Polon. Sci. Ser. Sci. Math. Astronom. Phys. 18 (1970), 371-374.

[18] A. Lasota and Z. Opial, An application of the Kakutani-Ky-Fan theorem in the theory of ordinary differential equations, Bull. Acad. Polon. Sci. Ser. Sci. Math. Astronom. Phys. 13 (1965), 781-786.

[19] B. Liu, Controllability of impulsive neutral functional differential inclusions with infinite delay, Nonlinear Anal. 60 (2005), no. 8, 1533-1552.

[20] T. W. Ma, Topological degrees for set-valued compact vector fields in locally convex spaces, Dissertationess Math. 92 (1972), 1-43.

[21] S. K. Ntouyas and P. Ch. Tsamatos, Global existence of semilinear evolution equations with nonlocal conditions, J. Math. Anal. Appl. 210 (1996), 679-687.

[22] A. Pazy, Semigroups of Linear Operators and Applications to Partial Differential Equations, Springer-Verlag, New-York, 1983.

[23] M. D. Quinn and N. Carmichael, An introduction to nonlinear control problems using fixed point methods, degree theory and pseudo-inverse, Numer. Funct. Anal. Optim. 7 (1984/1985), 197-219.

[24] A. M. Samoilenko and N. A. Perestyuk, Impulsive Differntial Equations, World Scientific, Singapore, 1995.

[25] H. Schaefer, Uber die method der a priori Schranken, Math. Anal. 129 (1955), 415-416.

[26] C. C. Travis and G. F. Webb, Cosine families and abstract nonlinear second-order differential equations, Acta Math. Acad. Sci. Hungar. 32 (1978), no. 1-2, 75-96.

[27] _ Second order differential equations in Banach spaces, Proc. Int. Symp. on Nonlinear Equations in Abstract spaces, 331-361, Academic Press New-York, 1978.

[28] R. Triggani, Addendum : A note on lack of exact controllability for mild solution in Banach spaces, [SIAM J. cont. Opti. 15 (1977), 407-411]; SIAM J. Cont. Opti. 18 (1980), no. 1, 98-99.

[29] K. Yosida, Functional Analysis, 6th Edition, Springer-Verlag, Berlin, 1980.

Dimplekumar N. Chalishajar

Department of Mathematics and Computer Science

Virginia Military Institute (VMI)

417, Mallory Hall, Lexington, VA-24450, USA

E-mail address: dipu17370@yahoo.com, chalishajardn@vmi.edu

FALGUni S. ACHARYA

Department of Applied Sciences and Humanities

Institute of Technology and Management (ITM) Universe

VADODARA-391510, GUJARAT, INDIA

E-mail address: falguni_69@yahoo.co.in 